\title{
VALUATION AND RETURN \\ DYNAMICS OF NEW VENTURES
}

\author{
Jonathan B. Berk \\ Richard C. Green \\ Vasant Naik
}

Working Paper 6745

http://www.nber.org/papers/w6745

\section{NATIONAL BUREAU OF ECONOMIC RESEARCH 1050 Massachusetts Avenue \\ Cambridge, MA 02138 \\ October 1998}

We wish to thank Lorenzo Garlappi, Walter Novaes, Bryan Routledge, Jeff Tarlowe, and Tan Wang for valuable comments and suggestions. We acknowledge gratefully research support from the QGroup, the Dean's Research Fund and the Center for Financial Markets at Carnegie Mellon (Green), and the Entrepreneurship Research Alliance at UBC and SSHRC (Naik). The views expressed here are those of the authors and do not reflect those of the National Bureau of Economic Research.

(C) 1998 by Jonathan B. Berk, Richard C. Green, and Vasant Naik. All rights reserved. Short sections of text, not to exceed two paragraphs, may be quoted without explicit permission provided that full credit, including $(\mathcal{C}$ notice, is given to the source. 
Valuation and Return Dynamics of New Ventures

Jonathan B. Berk, Richard C. Green, and Vasant Naik

NBER Working Paper No. 6745

October 1998

JEL No. G31, G12, G24, D92

\begin{abstract}
We develop and analyze a model of a multi-stage investment project that captures many features of $R \& D$ ventures and start-up companies. An important feature these problems share is that the firm learns about the potential profitability of the project throughout its life, but that "technical uncertainty" about the research and development effort itself is only resolved through additional investment by the firm. In addition, the risks associated with the ultimate cash flows the firm realizes on completion of the project have a systematic component, while the purely technical risks are idiosyncratic. Our model captures these different sources of risk, and allows us to study their interaction in determining the risk premia earned by the venture during development. Our results show that the systematic risk, and the required risk premium, of the venture are highest early in its life, and decrease as it approaches completion, despite the idiosyncratic nature of the technical risk.
\end{abstract}

Jonathan B. Berk

Haas School of Business

University of California

545 Student Services Bld

Berkeley CA, 94720-1900

and NBER

berk@u.washington.edu

Vasant Naik

Faculty of Commerce

University of British Columbia

2053 Main Mall

Vancouver, BC V6T 1 Z2

vasant@finance.commerce.ubc.ca
Richard C. Green

Graduate School of Industrial Administration Carnegie Mellon University

Pittsburgh, PA 15213

rg0b@andrew.cmu.edu 


\section{Introduction}

The valuation of ventures that produce cash flows only after multiple stages of development is an important problem for financial analysis. Within established organizations, managers are under increasing pressure to quantitatively justify expenditures on R\&D. Financial intermediaries, such as venture capitalists, routinely allocate capital resources on the basis of implicit or explicit valuations of firms with no assets, or few assets, other than the potential to generate cash flows in the future. Such firms are a growing presence on organized equity markets as well.

$R \& D$ ventures are subject to several, qualitatively different sources of risk. There is the "technical" uncertainty associated with the success of the research itself, or the time and cost required to bring it to fruition. Examples are the clinical trials of a new drug, or exploratory drilling for oil. There is the exogenous risk associated with the actions of a competitor or changes in the environment before or soon after product introduction. For example, a new drug may be rendered unnecessary by a superior treatment option. A software product may fail because of technological advances in hardware. Finally, there are the more traditional risks associated with demand for the product and production costs.

How do these types of risk jointly determine the value of an $R \& D$ venture? How much systematic versus unsystematic risk is borne by investors in $R \& D$, and how does this influence the risk premium they require? In this paper we develop a model that allows for all three types of uncertainty, and is still sufficiently tractable to provide qualitative and quantitative guidance regarding these questions.

Textbooks in corporation finance, in response to the tendency of practitioners and students to confuse systematic and unsystematic risks, often point to the technical risks of R\&D as examples of diversifiable risks that do not require compensation. Brealey and Myers (1996), for instance, list the risk of a dry hole in oil exploration, and denial of approval for a drug that cures baldness by the Food and Drug Administration as examples of "bad outcomes," that "appear to reflect unique (i.e., diversifiable) risks which would not affect the expected rate of return demanded by investors" (p. 220, their parentheses). Brealey, Myers and Marcus (1995), again considering the example of oil exploration, argue "... for the company as a whole, it's the average success rate that matters. Geological risks (is there oil or not?) should average out. The risk of a worldwide drilling program is much less than the apparent risk of any single wildcat well" (p. 237, their emphasis and parentheses). They go on to say that the investors in an oil company's stock will "... naturallyand realistically - assume that your successes and failures in drilling oil wells will average out with the thousands of independent bets made by the companies in their portfolio" (p. 237).

These arguments might be construed to imply that R\&D projects would not require a particularly high expected return. In our model we value simultaneously a multi-stage R\&D project and the stream of cash flows it will deliver if successful. We find the required risk premium for the $R \& D$ is higher than that of the underlying cash flows, and that the risk premium decreases as the project approaches completion. This behavior emerges despite the idiosyncratic nature of the technical risk associated with completing the $\mathrm{R} \& \mathrm{D}$. We argue that reasoning based simply on the idiosyncratic 
nature of purely technical risk ignores an important feature of actual R\&D projects. Decisions to continue with $R \& D$ are made conditioning on the resolution of systematic as well as unsystematic uncertainty. For example, while "geological risks" are arguably diversifiable, the decision to continue drilling at any particular site, as each stage of the development process is completed and technical uncertainty is resolved, is made with the current price of oil in mind. This imparts to the project the characteristics of a compound option. Options, because of the implicit leverage they impart, have higher systematic risk than the underlying asset.

In our model the firm consists of a single $R \& D$ project. When the $R \& D$ is successfully completed, the firm will generate a stream of stochastic cash flows, which we model as a geometric random walk that has both idiosyncratic and systematic risk. In order to realize these cash flows, however, the firm must first complete $N$ stages of $\mathrm{R} \& \mathrm{D}$. In this process, the venture is subject to four types of risk.

1. Technical risks pertaining to the successful completion of the R\&D itself: At each date an investment is required in order to keep the project "active." If the investment is made, then with some probability the current stage will be successfully completed and the firm can move on to the next stage. The event of success or failure at each stage is idiosyncratic risk.

2. The risk of competitive threat or obsolescence: We assume that with a fixed probability in each period the cash flows from the project, whether actual or potential, are extinguished. The uncertainty regarding obsolescence is idiosyncratic.

3. Uncertainty associated the potential future cash flows the project will produce if completed: At any point in time the firm observes the cash flows the project would be producing if it were already complete, and this state variable has both systematic and idiosyncratic risk.

4. Learning by doing: The probability of success at each stage is unknown to the firm's management, who, based on past outcomes, update their beliefs about it. Uncertainty about the duration and cost of the $R \& D$ is thus resolved by making investments in the process and observing the outcomes. The resulting expected cost to completion for the R\&D evolves endogenously, reflecting optimal policies.

In making its decisions about whether to continue investing, "mothball" the project, or simply abandon the venture, the firm observes state variables summarizing each of the underlying sources of uncertainty above: (1) the number of stages of R\&D still to be completed, (2) whether the project's potential cash flows have been extinguished through obsolescence, (3) the level of cash flow the project would be earning if the R\&D were complete, and (4) the history of its past attempts at completion.

We are able to obtain a number of interesting results on the interaction of these different sources of risk in determining the value and expected return on the venture. Perhaps most surprising is the role the resolution of idiosyncratic uncertainty plays in determining the current risk premium. As technical (idiosyncratic) uncertainty is resolved, the probability of successful and timely completion 
changes. This changes the properties of the option to mothball the project, which is exercised with the potential future cash flows in mind. Thus, although there is no risk premium earned on idiosyncratic risk per se, the resolution of idiosyncratic uncertainty can dramatically alter the risk premium earned on the venture as a whole.

We are also able to describe upper and lower bounds on the risk premium of the $R \& D$, and thus on the cost of capital of "pure growth" firms. We derive an analytically straightforward upper bound on the risk premium. The risk premium of the R\&D is close to this upper bound when the project is mothballed, generally early in its life. We show numerically that the risk premium of the $\mathrm{R} \& \mathrm{D}$ will not be lower than the premium earned on the cash flows from the project on completion. The risk premium on the $R \& D$ project falls from the upper to the lower bound over its life. This decline in the risk premium is non-linear, and is particularly pronounced around the time the firm just begins active development.

Learning by doing also plays an important role in determining the patterns of returns earned over the life of the project. As a result of learning, and endogenous policy responses to it, early resolution of technical uncertainty has a much greater impact on value and expected return than technical success later in the project's life. The firm will tolerate only a certain number of failures at a given stage of development before it abandons the $R \& D$. This threshold is quite low in the early stages, so it is quite possible for two identical firms to work on identical projects with very different results. One firm might be lucky, in that it achieves positive realizations of uncertainty in the early stages and ultimately completes the project. The other firm, because of bad luck early on, optimally decides to abandon the project and never learns about its underlying profitability.

Through simulation we are able to describe the properties of realized returns. We show that the option to abandon combines with the technological uncertainty to produce bimodal distributions, as one might expect of high risk, technological ventures. The expected cash flows from a typical project show the life cycle effects that characterize new products or industries.

While simple, our model of $\mathrm{R} \& \mathrm{D}$ is also flexible. Stages of different length, or with asymmetric probabilities of success, can to some extent be modeled as sequences of stages. We obtain closedform solutions for some special cases, which provide a benchmark against which to evaluate the numerical solutions we study for the general model. We are also able to prove a number of results characterizing solutions to the more general cases, even where we are unable to solve in closed-form for optimal policies and values.

In the next section we discuss other papers in the literature related to ours. Section 3 introduces the notation and describes the structure of the model. Section 4 then presents analytical results concerning the firm's optimal investment policies, the valuation of the R\&D venture, and the required risk premium it earns. Properties of these variables, and particularly of the risk premium, are studied numerically in Section 5, which also evaluates the distribution of realized returns. Section 6 concludes. More technical derivations and the proofs to the propositions in the paper are contained in the Appendix. 


\section{Review of Related Literature}

A large economics literature studies dynamic research and development policies for individual firms. (See, for example, Dutta (1997), Grossman and Shapiro (1986), Pakes (1986), Posner and Zuckerman (1990), Weitzman (1979), or Weitzman, Newey and Rabin (1981).) Another line of research evaluates socially optimal levels and allocations of investment in research, and the effects of patent law and policy. (See, for example, Bhattacharya and Mookherjee (1986), Dasgupta and Maskin (1987), and Gruver (1991).) The focus of all these models, however, is on optimal policies rather than on valuation. They typically assume, for example, risk neutrality or a concave utility function for the firm. This precludes a study of the determinates of systematic risk and risk premia, and their role in valuation, which is what we focus on.

More recently, a number of papers have studied the $R \& D$ process as a contingent claim on the value an underlying asset, which is interpreted as the present value of the cash flows on completion of the $R \& D$. This approach allows for richer specifications than more traditional dynamic programming solutions, and is closer in spirit to our paper. The valuation method in these papers differs from ours, however. They take as exogenous a stochastic process for the benefit or value of the project to the firm on completion of development. This evolves as a diffusion, and the project is valued as a contingent claim on this "underlying security." We take as exogenous the process describing the cash flows the project will generate, and value these cash flows and the R\&D project simultaneously. By deriving the value of the underlying cash flows rather than specifying it exogenously, we are able to focus on the relative systematic risk of $R \& D$ and the underlying cash flows and explain the dynamics of the risk premium of the R\&D.

Majd and Pindyck (1987) solve an investment problem in which the project requires a fixed total investment to complete, with a maximum instantaneous rate of investment. All the uncertainty in their model comes from stochastic evolution of the value of the project upon completion. There is no technical risk. The paper focuses on how the decision to invest or wait varies with the parameters and the single state variable in the model.

Childs and Triantis (1997) develop and numerically implement a model of dynamic R\&D that highlights the interactions across projects. They solve for and interpret optimal policies for a firm with multiple R\&D projects, which can run in parallel or sequentially, and calculate the values of the real options such problems present. Their model differs from ours both in function and in purpose. The richness of their specification allows them to analyze in detail the intensity and timing of optimal investment policies. The range of policies we allow for is more limited. The probalistic structures of the two models differ. Investment deterministically yields progress through investment stages in their model, where as in our model success is random. The information flows in the two models are also different. In Childs and Triantis (1997) the firm learns about the underlying value only if it invests. Otherwise the diffusion coefficient in the value process is set equal to zero, and the value "holds still." In our model, investing resolves technical uncertainty. Information about the potential cash flows continues to evolve independently of the firm's investment policy. 
Schwartz and Moon (1995) also use continuous-time methods to numerically value R\&D as a contingent claim on the value of the benefits created. Their paper is closest to ours in the sense that it allows simultaneously for three types of uncertainty. There is "technical" uncertainty associated with the success of the R\&D process itself. There is a an exogenous chance for obsolescence, during and after the development process, and there is uncertainty about the value of the project on completion of the R\&D. Schwartz and Moon (1995) solve for optimal investment policies, provide comparative statics regarding the option component of the project's value, and compare the project's value to the "NPV approach" which simply discounts expected cash flows given optimal policies. They take the value of the project on completion to be exogenous, however, and do not evaluate the relative systematic risk of $R \& D$ and the underlying cash flows. They also model technical uncertainty differently than we do. In their model, the expected cost to completion of the project is an exogenous stochastic process with drift and diffusion coefficients that depend on its current value and on the current level of investment. Given this process, optimal investment policies are obtained, and given those policies the expected cost to completion of the project may not have the functional form exogenously postulated. Our model takes as exogenous the technology for randomly advancing through stages of the project, and then derives optimal investment policies. These will lead to an endogenous process for expected cost to completion, which will have the desirable features of the process assumed by Schwartz and Moon (1995) (as well as Pindyck (1993)). Specifically, in our model innovations in the conditional expectation of remaining time to completion are zero when investment is zero, so there is learning by doing.

\section{The Model}

\subsection{Technology}

Consider a firm working on an R\&D project to bring a new product, process or technology to the market. Successful completion of the project involves completing $N$ stages, where $N$ is an exogenously given integer specific to the project. If and when it is completed, the project will generate a cash flow stream. These cash flows are subject to two types of shocks. There are persistent shocks to the level of the cash flow each period, modeled through a random walk. There is also a chance of a catastrophic event (like a competitor developing a superior product) that extinguishes the cash flow from the project forever. These shocks are realized whether the cash flows are potential, because the $\mathrm{R} \& \mathrm{D}$ has yet to be completed, or actual, because all $N$ stages have been passed.

We model the cash flows from the project with two stochastic processes $\{\delta(t), x(t), t \in \mathcal{T}\}$, where $\mathcal{T}$ denotes the set of positive integers. The process $\{x(t), t \in \mathcal{T}\}$ tracks whether a catastrophic event, such as the appearance of a product superior to the one being developed, has occurred. If $x(t)=0$

for any $t \in \mathcal{T}$ then the catastrophic event has occurred. If $x(t)=1$, it has not. We refer to the 
catastrophic event as "obsolescence." Assume that

$$
x(t+1)=x(t) y(t+1)
$$

where, for all $t \in \mathcal{T}, y(t+1)$ is independent of the past and independent of all other random variables in the model. The variable $y(t)$ is assumed take value 1 (when no castastrophic event occurs) with probability $\pi_{y}$ and a value 0 (when a catastrophic event does occur) with probability $1-\pi_{y}$.

The process $\{\delta(t), t \in \mathcal{T}\}$ specifies the cash flow conditional on no obsolescence. At any date $t$, while the R\&D is ongoing, $\delta(t) x(t)$ measures the current cash flow the project would have produced if it were completed. Before the $R \& D$ project is complete, therefore, the process $\{\delta(t)\}$ models the periodic arrival of information about the potential cash flows from the venture. If the $R \& D$ is successfully completed on date $t$ then for all $\tau \geq t, \delta(\tau) x(\tau)$ measures the actual cash flows from the project.

We assume that $\delta(t)$ evolves over time as follows:

$$
\delta(t+1)=\delta(t) \exp \left[\left(\mu-\frac{1}{2} \sigma_{\delta}^{2}\right)+\sigma_{\delta} \epsilon_{\delta}(t+1)\right]
$$

where $\left\{\epsilon_{\delta}(t), t \in \mathcal{T}\right\}$ is an independently and identically distributed collection of standard normal random variables.

At each date before the project is complete, the firm must decide whether to continue working on the project in an attempt to reach the next stage. To do so, it must incur a development cost of $I(t)$. This can be thought of as the salary to be paid to the research scientists and development engineers or the cost of research equipment, data, market research and analysis. The development costs can have both a fixed and a random, variable component:

$$
I(t)=a+b \delta(t) x(t)
$$

We allow the possibility for costs to have a proportional component because development costs are likely to be influenced by such factors as inflation and wage rates, for example. It also captures, to some extent, the practice of basing some part of employees compensation on an equity stake in the outcome of the R\&D. At times we will consider special cases where $a$ or $b$ equals zero to obtain tractability, or focus on particular aspects of the problem.

\subsection{Learning}

If, at any stage $n<N$ the firm makes the required investment, then we assume that with probability $\pi$ it succeeds and moves on to the next stage. With probability $1-\pi$, it makes no progress and stays where it was. The parameter $\pi$ is thus a measure of how productive the firm's R\&D activities are. We will consider cases where this is known to the firm, and also cases where the firm learns 
about $\pi$ by observing the success or failure of its previous efforts.

When the probability of success is not known, the success or failure of each effort the firm makes on the project conveys information about the unknown probability of success. To model this, we assume that the initial prior distribution of the probability of success at each stage, $\pi$, is a beta distribution with parameters $\left(\alpha_{1}, \alpha_{2}\right)$. The distribution of $\pi$, the probability of success, is updated according to Bayes' rule using the history of past attempts to complete all the various stages of research. If, at a particular date $t$, the firm has been successful in $n$ attempts out of $m+n$ trials, the firm's posterior distribution of $\pi$ on date $t$ is beta with parameters $\left(\alpha_{1}+n, \alpha_{2}+m\right)$. If the firm chooses to continue the research effort at $t$, at $t+1$ it will find out if it either succeeded or failed. If the firm succeeds, Bayes' rule implies that the posterior distribution of $\pi$ at date $t+1$ will be beta with parameters $\left(\alpha_{1}+n+1, \alpha_{2}+m\right)$. If the firm fails, the posterior distribution of $\pi$ at $t+1$ is beta with parameters $\left(\alpha_{1}+n, \alpha_{2}+m+1\right){ }^{1}$

\subsection{Valuation Processes}

To analyze the value of an asset whose cash flows are uncertain, and understand its dependence on its systematic risk, we must put structure on the pricing kernel in the economy. Let the pricing kernel be denoted $\{z(t), t \in \mathcal{T}\}$. That is to say, the date $t$ value of any cash flow arriving at $T$ is computed as

$$
E_{t}\left[\frac{z(T)}{z(t)} C(T)\right]
$$

and covariance with the pricing kernel can be interpreted as systematic risk, corresponding, for example, with "beta" under the mean-variance CAPM.

We assume that the pricing kernel evolves through time as follows:

$$
z(t+1)=z(t) \exp \left[-r-\frac{1}{2} \sigma_{z}^{2}-\sigma_{z} \epsilon_{z}(t+1)\right]
$$

where $r$ denotes the one-period riskless rate, and $\left\{\epsilon_{z}(t), t \in \mathcal{T}\right\}$ is an independently and identically distributed collection of random variables. We assume the riskless rate is constant. This is done to focus on risk premia that are attributable to systematic risk that, in our setting, is analogous to "market risk" rather than interest rate risk.

Finally, we assume that for any $t$, the pair of innovations, $\left\{\epsilon_{\delta}(t), \epsilon_{z}(t)\right\}$, has a bivariate normal distribution, where the means of both variables are zero, their variances are one, and their covariance is $\rho$. We denote the market price of risk as $\lambda \equiv \rho \sigma_{\delta} \sigma_{z}$. This parameter determines the equilibrium excess return earned by holding an equity position in the underlying cash flow process, $\delta(t)$. It thus provides a benchmark against which to evaluate the risk premia earned by holding an equity stake

\footnotetext{
${ }^{1}$ These conclusions are based on the following fact: suppose, $X_{i}, i=1, \ldots, n$ is a random sample from a Bernoulli (binomial) distribution with an unknown parameter value $W$. Suppose that the prior distribution of $W$ is a beta distribution with parameters $\alpha_{1}>0$ and $\alpha_{2}>0$. Then, the posterior distribution of $W$, when $X_{i}=x_{i}, i=1, \ldots, n$, is a beta distribution with parameters $\alpha_{1}+y$ and $\alpha_{2}+n-y$ where $y=\sum_{i=1}^{n} x_{i}$. See Theorem 1 in Section 9.2 of DeGroot (1970).
} 
in the $\mathrm{R} \& \mathrm{D}$ project.

It would be straightforward, although algebraically and computationally burdensome, to generalize the model to allow for stochastic interest rates that follow a simple one-factor model, as in Berk, Green and Naik (1997). Assuming interest rates are constant and innovations to the pricing operator are independent and identically distributed through time has some expositional advantages for our problem. It ensures that risk premia are constant for the underlying cash flow process, and that all of the state variables we must track in valuing the firm and determining optimal policies are firm specific. Thus, any variability in the risk premia can be attributed to the special features of the $R \& D$ venture rather than movements in the economy in general. In addition, these assumptions allow for simple, parametric decompositions between systematic and unsystematic risk. In this way the model can provide qualitative guidance about how the characteristics of R\&D projects should influence judgments about required rates of return.

\section{Valuation}

We begin by briefly summarizing the firm's decision problem. At any date $t$, the firm in our model must decide whether to invest in additional R\&D or not. The firm solves this discrete choice problem to maximize its current market value. The cost of continuing is the additional investment required for the current period, $I(t)$. The benefit is the chance to move ahead through one of the $N$ stages required to complete the project, and thus gain control of the periodic cash flows given by the process $\{\delta(t), t \in \mathcal{T}\}$. The probability that the firm will move ahead a stage, if it makes the investment, is given by $\pi$, a parameter the firm evaluates through Bayes' rule on the basis of past successes and failures of its $R \& D$ efforts. If the firm does not invest, its information set and value will remain fixed except for the exogenous evolution of $\delta(t)$.

Thus, the state variables on which the firm must base its decision at $t$ are as follows:

$m(t)$ The number of previous investments that have failed to move the venture through a stage of the $R \& D$ process.

$n(t)$ The number of stages of the $\mathrm{R} \& \mathrm{D}$ process the venture has successfully passed through. The number of remaining stages is $N-n(t)$.

$\delta(t)$ The current or potential cash flow from the project.

$x(t)$ An indicator equal to zero when the project is obsolete, and otherwise one.

Let $V[n(t), m(t), \delta(t)]$ be the current value of the firm conditional on $x(t)=1$. If $x(t)=0$, the value of the venture is zero. It is also apparent that if $n(t)=N$, the project is complete and $V(N, m, \delta)$ will just be the present value of future cash flows regardless of $m$. 


\subsection{Valuation of the Cash Flow Process}

We can compute the value of the cash flow process at any date as

$$
E_{t}\left\{\sum_{s=0}^{\infty} \frac{z(t+s)}{z(t)} \delta(t+s) x(t+s)\right\}
$$

Note this is the "cum-dividend" value, as it includes the current cash flow. This will coincide with the value of the firm or venture when the $R \& D$ is complete. Our distributional assumptions give the value of the cash flows a very simple form.

Proposition 1 The value of the completed project is

$$
V[N, m(t), \delta(t)]=\frac{\delta(t)}{1-\pi_{y} e^{\mu-(r+\lambda)}}
$$

This expression is recognizable as the continuously compounded version of the growing perpetuity formula. Here the discount rate is the sum of the riskless rate and the risk premium. The fact

that the cash flows terminate stochastically is captured by $\pi_{y}$ in the denominator. For convenience we will henceforth define this discount factor to be

$$
\beta \equiv \pi_{y} \exp [\mu-(r+\lambda)]
$$

The role of systematic risk in reducing the value of the cash flows is evident in the presence of $\lambda$ in the expression for $\beta$, where it augments the riskless rate in discounting the cash flows. For the value to be finite, it is obvious that $\beta$ must be a fraction. In our expression for the pricing kernel, (5), the innovations enter negatively, so that higher $\lambda$ corresponds to more (undesirable) systematic risk. The fact that the systematic risk enters the valuation through a multiplicative constant ensures that the expected return to holding an equity position in the completed project is constant.

Proposition 2 The per-dollar continuously compounded expected return on a security that pays the stream $\{\delta(t), t \in \mathcal{T}\}$ is constant and equal to $r+\lambda$.

\subsection{The Value Function}

In the appendix we construct the value function for the firm's problem of deciding whether to invest in R\&D. This function serves both as the objective of the firm's dynamic programming problem, and as the market value of the $R \& D$ project. 
To construct this function note that the value today is just the expected (discounted) value of the function in one period. This expectation must be taken over all possible states. If the firm makes the investment, these will be the discrete outcomes associated with obsolescence or the lack of it, and failure or success in moving through the current stage. In addition there are the infinitely many states corresponding to the different possible values for $\pi$ and $\delta(t+1)$. If the firm does not invest, then the expectation will only be over the states corresponding to possible obsolescence and $\delta(t+1)$. The decision on whether or not to mothball the project is made to maximize value, thus, for $n<N$, the value function must satisfy the following recursion:

$$
\begin{aligned}
& V(n, m, \delta)= \\
& \quad \max \left\{\int_{0}^{1}\left[\pi V_{e}(n+1, m, \delta)+(1-\pi) V_{e}(n, m+1, \delta)\right] v(\pi \mid m, n) d \pi-a-b \delta, V_{e}(n, m, \delta)\right\}
\end{aligned}
$$

where $v(. \mid n, m)$ denotes the conditional distribution of $\pi$ given that the firm has made $n$ successful and $m$ unsuccessful attempts thus far, and $V_{e}(n, m, \delta)$ is the current discounted value of the firm next period conditional on: (1) being currently alive and (2) holding fixed its progress in R\&D at $n$ stages complete over $n+m$ trials. That is,

$$
\begin{aligned}
& V_{e}(n, m, \delta)= \\
& \quad E_{t}\left\{\frac{z(t+1)}{z(t)} x(t+1) V[n, m, \delta(t+1)] \mid \delta(t)=\delta, x(t)=1, n(t)=n, m(t)=m\right\} .
\end{aligned}
$$

Note that the $\max \{\cdot, \cdot\}$ operator in (9) implements the decision on whether to invest in the current period. If the investment is made, the expected value of the project in one period reflects both whether the next stage is successfully completed and the updating that occurs to the probability of success. If the project is mothballed, then its expected value does not depend on either.

In the appendix, we first show that the recursion (9) has a unique solution. We then consider the model with a finite-time horizon for completion of the $\mathrm{R} \& \mathrm{D}$. That is, if the $N$ stages are not finished by some date $T$, the project becomes worthless. We verify that the solution to the recursion in (9) is the limit of the value of the R\&D project as $T \rightarrow \infty$, and thus that it is, indeed, the value of the project for a firm facing an infinite horizon.

Since the integrand inside the $\max \{\cdot, \cdot\}$ operator on the right-hand side of (9) is linear in $\pi$, the above recursion can be restated as:

$$
\begin{aligned}
& V(n, m, \delta)= \\
& \quad \max \left\{\bar{\pi}(n, m) V_{e}(n+1, m, \delta)+(1-\bar{\pi}(n, m)) V_{e}(n, m+1, \delta)-a-b \delta, V_{e}(n, m, \delta)\right\}
\end{aligned}
$$

where

$$
\bar{\pi}(n, m)=\int_{0}^{1} \pi v(\pi \mid n, m) d \pi=\frac{\alpha_{1}+n}{\alpha_{1}+\alpha_{2}+n+m},
$$

is the mean of the firm's posterior distribution over $\pi$. 
We next establish a number of general properties the value of the $R \& D$ venture must satisfy.

Proposition 3 The value of the RESD venture, $V(n, m, \delta)$,

1. is monotone and convex in $\delta$, the value of the cash flows

2. is increasing in $n$, the number of stages completed, and decreasing in $m$, the number of failures

3. approaches zero as $\delta$, the potential cash flow, approaches zero

This proposition shows that the value of the venture responds in predictable ways to the state variables $\delta, n$ and $m$. Successfully passing through $R \& D$ stages is good news. Intuitively, a higher $n$ only relaxes constraints on the firm in its goal of realizing cash flows. Similarly, higher failure rates are bad news. The firm's posterior assessment of its chances of ever realizing the cash flows will decrease as $m$ increases, holding $n$ fixed. The monotonicity and convexity in $\delta$ follows directly from the value function's option-like properties. Since the value of the firm is always the expected discounted value of its future cash flows, its value must approach zero as these cash flows approach zero.

\subsection{Proportional R\&D Costs}

The case with no fixed costs $(a=0)$ is interesting for several reasons. A closed-form (up to a finite summation) solution for the value is attainable so that properties of the optimal investment policy can be easily established and intuitively evaluated. This investment policy has a very simple form. The firm will never "mothball" the project in hopes that conditions will improve and lead to a resumption of development. It will only abandon the project. This abandonment decision is driven entirely by learning about the firm's research productivity $(\pi)$. These results will prove important when we return to the general case in Section 4.4. The solution in the proportional cost case is also required to establish some of the properties of the general solution. It will also be used informally as a benchmark to evaluate the incremental effects of the decision to mothball.

The tractability of this case derives from the fact that the value function is proportional to the level of the cash flow process.

Proposition 4 If $I(t)=b \delta(t)$ then the value function is homogeneous of degree one in $\delta$, and we have

$$
V(n, m, \delta)=\delta h(n, m)
$$

and

$$
V_{e}(n, m, \delta)=\delta \beta h(n, m),
$$

with $\beta=\pi_{y} \exp [\mu-(r+\lambda)]$. The function $h(n, m)$ satisfies the recursion:

$$
h(n, m)=\max [\bar{\pi}(n, m) \beta h(n+1, m)+(1-\bar{\pi}(n, m)) \beta h(n, m+1)-b, \beta h(n, m)]
$$


To obtain and expression for $h(n, m)$, we must first derive appropriate boundary conditions. Then we will show that the optimal investment strategy responds to experience in a uniform way — the firm only abandons when the number of failures reaches some threshold which we call $m^{*}(n)$. This allows us to substitute this upper bound on past failures for the $\max [\cdot, \cdot]$ operator, and derive a solution through recursive substitution. The following propositions accomplish these tasks.

Proposition 5 When $I(t)=b \delta(t),(a=0, b>0)$ moth-balling is equivalent to abandonment, and if $h(n, m)$ solves (15) it also solves

$$
h(n, m)=\max [\bar{\pi}(n, m) \beta h(n+1, m)+(1-\bar{\pi}(n, m)) \beta h(n, m+1)-b, 0] .
$$

Furthermore, $h(N, m)=\frac{1}{1-\beta}$ for all $m$, and for every $n<N$ there must exist an integer $m^{*}(n)<\infty$ such that for all $m>m^{*}(n), h(n, m)=0$.

The finiteness of $m^{*}(n)$ and the value of the $\mathrm{R} \& \mathrm{D}$ upon completion provide the necessary boundary conditions. By the definition of $m^{*}(n), h(n, m)>0$ for all $m \leq m^{*}(n)$. So for all $m \leq m^{*}(n)$,

$$
h(n, m)=\bar{\pi}(n, m) \beta h(n+1, m)+(1-\bar{\pi}(n, m)) \beta h(n, m+1)-b
$$

and

$$
h\left(n, m^{*}(n)\right)=\bar{\pi}\left(n, m^{*}(n)\right) \beta h\left(n+1, m^{*}(n)\right)-b .
$$

Thus, $m^{*}(n)$ can be computed as

$$
\max \{m \mid \bar{\pi}(n, m) \beta h(n+1, m)-b>0\}
$$

A closed-form expression for the functions $h(n, m)$ are computed recursively as follows. Suppose that we already have the solution to $h(n+1, \cdot)$. Once we have $m^{*}(n)$, we can compute the value of $h(n, m)$ for $m<m^{*}(n)$, simply by recursive substitution in (17). The following proposition states the results of this procedure.

\section{Proposition 6}

$$
h(n, m)=\left\{\begin{array}{l}
\frac{1}{1-\beta}, \text { if } n=N \\
0, \text { if } n<N \text { and } m>m^{*}(n) \\
\sum_{i=0}^{m^{*}(n)-m}\left[\beta\left(\frac{n+\alpha_{1}}{m+n+i+\alpha_{1}+\alpha_{2}}\right) h(n+1, m+i)-b\right] \beta^{i} \frac{\left(m+\alpha_{2}\right)_{i}}{\left(m+n+\alpha_{1}+\alpha_{2}\right)_{i}}, \text { o.w. }
\end{array}\right.
$$

where $m^{*}(n)$ is defined implicitly as the largest $m$ such that

$$
m<\frac{\left(n+\alpha_{1}\right) \beta h(n+1, m)}{b}-n-\alpha_{1}-\alpha_{2} .
$$


and $(\alpha)_{n} \equiv \frac{\Gamma(\alpha+n)}{\Gamma(\alpha)}$ is the Pochhammer symbol.

The next step is to use the other boundary condition that $h(N, m)=1 /(1-\beta)$ in (20) to provide a closed-form expression for $h(N-1, \cdot)$. This expression can then be used in (20) to recursively obtain the comparable closed-form expression for all $n<N-1$.

When $\pi$ is known we can drop $m$, which summarizes the history past investment in the project, as a state variable. Define

$$
g(n) \equiv h(n, m),
$$

so that the value of the project is $g(n) \delta$. If $g(0) \leq 0$, the project is never started so $g(n)=0$, and we can limit attention to the case when $g(0)>0$. Proposition 3 , shows that the value is increasing in $n$, the number of stages passed through. Thus, in this case the project will never be abandoned. Substituting (22) into (17) provides:

$$
g(n)=\pi \beta g(n+1)+(1-\pi) \beta g(n)-b
$$

for $n=0,1, \ldots, N-1$. By Proposition 6 it must also be the case that

$$
g(N)=h(N, m)=\frac{1}{1-\beta} .
$$

The following proposition provides the solution to this recursion:

Proposition 7 When $I(t)=b \delta(t)$ and $\pi$ is known, the value of the RED venture at date $t$ given that it has attained stage $n$ is $\delta(t) g(n)$ where if

$$
g(0) \equiv\left[\frac{1}{1-\beta}+\frac{b \theta}{1-\gamma}\right] \gamma^{N}-\frac{b \theta}{1-\gamma}>0
$$

with

$$
\gamma \equiv \frac{\pi \beta}{1-(1-\pi) \beta}
$$

and

$$
\theta \equiv \frac{1}{1-(1-\pi) \beta}
$$

then

$$
g(n)=\left[\frac{1}{1-\beta}+\frac{b \theta}{1-\gamma}\right] \gamma^{N-n}-\frac{b \theta}{1-\gamma}
$$

and otherwise $g(n)=0$ for all $n \leq N$. Furthermore, the value of the project is increasing and convex in number of stages completed.

\subsection{Optimal Investment Policy}

The properties of the solution under proportional costs are useful for understanding the solution in the general case. For example, it is evident that the solutions for the general and the proportional 
case converge as the value of the cash flow grows and the "leverage" associated with the fixed costs becomes less important. The next proposition proves this.

Proposition 8 As $\delta(t)$ grows without bound, the value of the project approaches its value for the proportional case.

$$
\lim _{\delta \rightarrow \infty} \frac{V(n, m, \delta, 1)}{\delta}=h(n, m)
$$

The optimal policies for the proportional case also help to delineate tradeoffs that govern optimal policies in the general case. When costs are strictly proportional, while the option to suspend R\&D is valuable, once suspended the project is never restarted. Intuitively, this is because no learning about the firm's R\&D capabilities $(\pi)$ takes place without investment. Learning about the firm's cash flows, by observing $\delta(t)$, does not lead to a resumption of R\&D spending, because it effects both costs and benefits proportionally.

The other extreme is when costs have no proportional component. In this case, there is no reason to ever abandon the project. Once investment is suspended, learning about the firm's capabilities ceases, and learning about the value of the cash flows continues. There is always a chance that $\delta(t)$ will grow sufficiently large that it will render the fixed costs insignificant relative to the project's potential value.

Proposition 9 When $I(t)=a,(b=0)$ the firm always mothballs and never abandons the R\&SD project, while when $I(t)=b \delta(t)$, $(a=0)$ the firm always abandons and never mothballs the project.

Our next result establishes the optimal investment policy for the general case. We first establish that the firm will eventually give up in the face of repeated failure and exercise its abandonment option. To see this, note that for fixed $b$, the value of the project with $a=0$ must be greater than with $a>0$. Thus, since Proposition 5 shows that an $m$ exists when it is optimal to abandon when $a=0$, it must also be optimal to abandon at this $m$ when $a>0$. The proposition also proves that it is suboptimal to abandon before this $m$.

We also derive the optimal time to mothball the project. This requires a series of lemmas, stated and proved in the appendix, regarding the slopes of the arguments of the two terms in the recursion that gives the value function, equation (9). These arguments specify the anticipated value of the project given that investment continues, and its value when investment is suspended. The lemmas show that the value conditional on continued investment is always more responsive to the value of the underlying cash flows. Thus, for fixed $n$ and $m$ there is a unique value of $\delta(t)$ at which the firm is indifferent to investing or not. Optimal policies can be characterized in terms of this value. We exploit the fact that the critical value of $\delta(t)$ is increasing in the fixed cost in our numerical analysis. 
Proposition 10 (Optimal Investment Policy) When costs have both proportional and fixed components $(a>0, b>0)$, then for $m^{*}(n)$ defined in Proposition 6 , the project is abandoned if and only if $m>m^{*}(n)$. If $m \leq m^{*}(n)$, then there is a unique $\delta^{*}(n, m)$ such that the project is mothballed if and only if $\delta(t)<\delta^{*}(n, m)$. Furthermore, $\delta^{*}(n, m)$ is decreasing in a, so if the fixed costs are increased projects are mothballed at lower levels of $\delta(t)$.

There are two ways a project can be mothballed - either a negative resolution of uncertainty causes $\delta$ to drop below $\delta^{*}$, or repeated failure causes $\delta^{*}$ to rise above the current $\delta$. However, there is only one way a project can be abandoned-through repeated failure. A negative resolution of $\delta$ uncertainty cannot lead to abandonment because $m^{*}$ is not a function of $\delta$. Of course, once the project is abandoned its value drops to zero which implies that $\lim _{m->\infty} V(n, m, \delta)=0$.

\subsection{Risk Premia}

In this section we study the required expected return earned on an ownership claim to the $R \& D$ venture. We are interested both in how the systematic and idiosyncratic risks of the venture interact to determine this required return, and in the dependence of the required return on how close the project is to completion. It seems intuitive that a project close to completion should inherit to a greater degree the risk characteristics of the stochastic cash flow stream it will ultimately deliver. Those far from completion might be expected to behave quite differently.

This analysis may be helpful in applications where choices must be made about the discount rate or cost of capital appropriate for evaluating R\&D expenditures. Standard practice appears to be to apply a constant discount rate to "probability weighted" cash flows in calculating present values. The discount rate employed is generally the firm's estimated cost of capital, or a cost of capital appropriate to the industry in which the investment is being made.

Myers and Howe (1997) argue that the cost of capital should decrease through the life of the project, due to the higher "leverage" of the project early in its life. At first, much of the present value of the project is associated with the relatively fixed $R \& D$ investments, as opposed to the variable net revenues that begin when the project is finished. Analogous to a levered firm, the required return on the "equity" in the project should be higher when it is more levered. As the R\&D is brought to completion, and the fixed claims are "paid down," the required return should fall. Our model provides a natural laboratory in which to evaluate these intuitions and quantify their importance.

The model provides a particularly simple setting in which to address these questions because the risk premium earned by the underlying cash flow process is constant, as Proposition 2 shows. Thus, any variability in the required return must be due to characteristics of the R\&D itself.

Since we cannot solve explicitly for the value function and thus the risk premium, our ability to address these questions analytically is limited. We can learn a good deal about the sources of variation in the risk premium, however, by considering cases where it is constant. An incomplete project differs from a completed one for four reasons. First, the stream of cash flows is randomly 
delayed. Second, the firm incurs fixed costs prior to claiming the random cash flow stream. Thus, the firm is "short" a fixed claim and "long" a variable claim. This confers operating leverage on the project. Third, the firm holds an option to delay these expenditures. Fourth, it also has an option to abandon the project altogether. As Proposition 10 makes clear, the decision to abandon versus the decision to mothball can be viewed as qualitatively different in that the decision to abandon is made purely in response to technological (idiosyncratic) risk. The "cutoff rule" at which the project has zero continuation value is of the form $m^{*}(n)$, and does not depend on $\delta(t)$. The decision to suspend investment, on the other hand, depends on information about future cash flows, and these have systematic risk.

When costs are proportional, then the option to mothball (rather than abandon) has no value, and the leverage associated with the fixed component of the costs is absent. The next proposition shows that in this case the risk premium on the project is the same as that earned on the cash flows, and thus that the sources of variation in the risk premium are attributable to precisely these factors.

Proposition 11 When there are no fixed R\&D costs $(a=0)$ the risk premium of the venture is independent of $n$ or $m$, and equal to $\lambda$, the risk premium of the completed project.

A further specialization of the models allows us to ask what effect pure random delay has on risk premia in a capital budgeting setting. A special case of Proposition 11 is when all R\&D costs are zero, so that the "R\&D" is just a delay in the arrival of a stream of cash flows. Because the factors contributing to delay are purely idiosyncratic, one might think that the systematic risk of the $R \& D$ would be less than that of the cash flows themselves. Proposition 11 shows that this is not the case. Higher probability of delay reduces both the value today and the expectation of future value proportionally, as long as the delay is idiosyncratic, so that the expected return is unaffected. ${ }^{2}$

The fact that the decision to abandon does not affect the risk premium is not only a property of this special case, and it illustrates an important point. The factors that impart systematic risk to a venture are naturally viewed as exogenous to the $R \& D$ process itself. Information about these exogenous factors continues to evolve independent of the firm's investment choices. For example, macroeconomic conditions can be observed without direct investment by the firm. Thus, the response to bad news about such factors is to delay investment and see if conditions improve. The decision to walk away from a project is much more likely to be driven by a determination that it is technologically infeasible or prohibitively costly, and the factors leading to such a determination are likely to be idiosyncratic to the project, firm, or industry.

\footnotetext{
${ }^{2}$ By way of analogy, consider a lottery that, with a fixed probability, simply delivers the S\&P 500 to the winner at date $t+\tau$. The arguments in the proof of Proposition 11 tell us that this lottery will be priced at date $t$ to yield the same expected return as the S\&P. A more complicated lottery that offered different randomly sized positions in the $\mathrm{S} \& \mathrm{P}$, at a random future date, would also be priced this way. Since the variables that determine exercise of the options embedded in R\&D are exercised independently of $\{\delta(t), t \in \mathcal{T}\}$ when costs are proportional, the project's payoffs are similar to those of such a lottery.
} 
Our model illustrates these distinctions in a particularly stark manner. By Proposition 10, the decision to abandon the venture does not depend on $\delta$, that is, it is a function of only technological risk which is idiosyncratic. Thus, this option will never command a risk premium. By the same token, the optimal exercise of the option to mothball does depend on $\delta$ (by the same proposition), and so R\&D projects in which this option is valuable will command risk premium in excess of $\lambda$.

Of course, even though the firm's investment experience is idiosyncratic, it still affects the nature of the firm's current position in the systematic variable, and its exposure to this risk. Thus, in the general case, we find that optimal exercise of the option to mothball depends on $n$ and $m$. A change in either $n$ or $m$ will affect the "in the moneyness" of the option, and the risk premium of an option is affected by how much it is in or out of the money. This implies is that the amount by which the risk premium of the incomplete project exceeds $\lambda$ is affected by the resolution of technical uncertainty, even though this risk is purely idiosyncratic and so is not priced. Intuitively, the reason for this is that one can think of the total expected costs of the R\&D as the "strike" price of the growth option. Completing a stage reduces this expected cost, which lowers the strike price of the option and the risk premium.

A natural way to think of the $R \& D$ venture, then, is as a series of compound options on the underlying cash flows. This analogy suggests that when the option value is a function of systematic risk, the project will have higher required returns as we move back through its stages. We next show, numerically, that this is indeed the case.

\section{$5 \quad$ Numerical Results}

In this section we use numerical methods to analyze in greater detail some of the properties of the $R \& D$ venture. We consider two parameterizations. First, we calculate values and risk premia assuming that $\pi$ is known. This special case provides a simple environment in which the incremental importance of the option to mothball can be gauged. The second parameterization is the general model and allows an assessment of how the risk premium is affected by the resolution of uncertainty about the firm's research productivity. Since in the general model it might be optimal to abandon, this case also allows us to investigate why the option to mothball commands a risk premium, while the option to abandon does not.

We parameterize the model to quarterly data; a period corresponds to three months. Table 1 lists the values of the parameters employed. The project would take five years to complete if every investment were successful. The dividend process grows at $3 \%$ per year on average, with an annual standard deviation of about $70 \%$, so its innovations are quite variable relative to this growth rate. The firm's and the market's priors about the chance of success at each stage are diffuse and uniform, a special case of the beta with both parameters equal to one. Alternatively, when $\pi$ is known, success occurs at each stage with a probability of $50 \%$, which is also the initial prior in the unknown case. The probability of surviving one year without obsolescence $\left(\pi_{y}^{4}\right)$ is $90 \%$. The fixed costs are assumed to be 1.0 per period while the variable costs are 0.1 . Thus for values of $\delta$ around 


\begin{tabular}{lcc}
\hline Parameter & $\pi$ unknown & $\pi$ known \\
\hline $\mathrm{N}$ & 20 & 20 \\
$\mu$ & 0.0075 & 0.0075 \\
$\sigma_{\delta}$ & 0.2 & 0.2 \\
$r$ & 0.0175 & 0.0175 \\
$\sigma_{z}$ & 0.2 & 0.2 \\
$\pi$ & - & 0.5 \\
$\alpha_{1}$ & 1.00 & - \\
$\alpha_{2}$ & 1.00 & - \\
$\pi_{y}$ & 0.974 & 0.974 \\
$a$ & 1.0 & 1.0 \\
$b$ & 0.1 & 0.1 \\
$\lambda$ & 0.02 & 0.02 \\
$\phi \lambda$ & 0.05 & 0.05 \\
\hline
\end{tabular}

Table 1: Parameter Values for Simulations

ten the fixed and variable costs contribute equally to the total costs. The value of the standard deviation in the pricing kernel (about 7\% per year) is chosen, together with the correlation between the pricing kernel's innovations and those of the cash flow process (0.5) to provide a $\lambda=0.02$. On an annual basis this corresponds to a risk premium of $8 \%$ for the cash flows of the completed project. Since the risk free rate is $7 \%$ per year, this implies an expected return of $15 \%$ for the completed project. The final line in Table 1 gives the upper bound $(\phi \lambda)$ for the risk premium, derived below in Proposition 12. This is 0.05 or $20 \%$ on an annual basis, which implies an upper bound on the expected return of the incomplete project of $27 \%$ annually.

\subsection{Known Probability of Success}

The case where $\pi$ is known to the firm provides a useful benchmark for several reasons. In this case all uncertainty is attributable to just two sources, the characteristics of the cash flow and the pure uncertainty of overcoming technical hurdles. This allows us to analyze the effects that are not a function of the uncertainty about technical ability.

With proportional $\mathrm{R} \& \mathrm{D}$ costs, the option to mothball is never exercised and so the incomplete project has the same risk premium as the completed project. When there is a fixed component in the $R \& D$ cost, the option to mothball has value and commands a risk premium. The voluntary nature of the investment decision, which is made conditioning on current values of $\delta(t)$ imparts systematic risk to the project. In addition, the firm's fixed investment expenditures can be viewed as a liability that offsets the value of its asset, the cash flow stream. Since the fixed liability has lower risk than the stochastic asset, the firm has operating leverage that will also alter the risk premium. To gauge the relative importance of these two effect, we compute the value and expected return of the incomplete project with and without the option to mothball. That is, we compute 
the value of the project as in our model. We also compute it assuming the firm must invest each period. Closed form expressions for the value in this case are provided in Lemma 3, which is stated and proved in the Appendix.

Table 2 shows the value of the R\&D project for different levels of $\delta$ at different stages of development. The values in parenthesis is the value of the venture assuming investment is nonvoluntary. Thus, the difference between the actual values and the values in parentheses is a measure of how important the option, as opposed to just the operating leverage associated with the fixed costs, is. The value of this option is most important in the early stages of development and lower values of $\delta$. The importance of the convexity that this imparts can be gauged by comparing the price appreciation for an increase in $\delta$ when the option is important (e.g., 4 completed stages) to when it is not (e.g, 16 completed stages).

\begin{tabular}{|c|c|c|c|c|c|c|c|}
\hline \multirow[b]{2}{*}{$\delta$} & & \multicolumn{6}{|c|}{ Number of Completed Stages $(n)$} \\
\hline & & 0 & 4 & 8 & 12 & 16 & 20 \\
\hline \multirow{2}{*}{5} & Value & $\begin{array}{c}\mathbf{. 0 1} \\
(-17.06)\end{array}$ & $\begin{array}{c}\mathbf{5 2} \\
(-9.18)\end{array}$ & $\begin{array}{c}\mathbf{4 . 4 9} \\
(2.80)\end{array}$ & $\begin{array}{c}21.07 \\
(21.04)\end{array}$ & $\begin{array}{c}48.84 \\
(48.84)\end{array}$ & $\begin{array}{c}91.26 \\
(91.26)\end{array}$ \\
\hline & $\begin{array}{l}\text { Risk } \\
\text { Prem }\end{array}$ & $\begin{array}{c}0.200 \\
(-)\end{array}$ & $\begin{array}{c}\mathbf{0 . 2 0 0} \\
(-)\end{array}$ & $\begin{array}{c}\mathbf{0 . 1 9 8} \\
(0.330)\end{array}$ & $\begin{array}{c}0.116 \\
(0.117)\end{array}$ & $\begin{array}{c}0.089 \\
(0.089)\end{array}$ & $\begin{array}{c}0.080 \\
(0.080)\end{array}$ \\
\hline \multirow{2}{*}{10} & Value & $\begin{array}{c}\mathbf{. 0 8} \\
(-14.99)\end{array}$ & $\begin{array}{c}\mathbf{2 . 9 4} \\
(-0.94)\end{array}$ & $\begin{array}{c}20.72 \\
(20.60)\end{array}$ & $\begin{array}{c}53.66 \\
(53.66)\end{array}$ & $\begin{array}{c}104.45 \\
(104.45)\end{array}$ & $\begin{array}{c}182.53 \\
(182.53)\end{array}$ \\
\hline & $\begin{array}{l}\text { Risk } \\
\text { Prem }\end{array}$ & $\begin{array}{c}0.200 \\
(-)\end{array}$ & $\begin{array}{c}0.200 \\
(-)\end{array}$ & $\begin{array}{l}0.1265 \\
(0.129)\end{array}$ & $\begin{array}{c}0.095 \\
(0.095)\end{array}$ & $\begin{array}{c}0.084 \\
(0.084)\end{array}$ & $\begin{array}{c}0.080 \\
(0.080)\end{array}$ \\
\hline \multirow{2}{*}{15} & Value & $\begin{array}{c}\mathbf{0 . 2 2} \\
(-12.92)\end{array}$ & $\begin{array}{c}8.21 \\
(7.29)\end{array}$ & $\begin{array}{c}38.42 \\
(38.39)\end{array}$ & $\begin{array}{c}86.28 \\
(86.28)\end{array}$ & $\begin{array}{c}160.06 \\
(160.06)\end{array}$ & $\begin{array}{c}273.79 \\
(273.79)\end{array}$ \\
\hline & $\begin{array}{l}\text { Risk } \\
\text { Prem }\end{array}$ & $\begin{array}{c}0.200 \\
(-)\end{array}$ & $\begin{array}{c}0.171 \\
(0.211)\end{array}$ & $\begin{array}{c}0.107 \\
(0.107)\end{array}$ & $\begin{array}{c}0.089 \\
(0.089)\end{array}$ & $\begin{array}{c}0.083 \\
(0.083)\end{array}$ & $\begin{array}{c}0.080 \\
(0.080)\end{array}$ \\
\hline \multirow{2}{*}{20} & Value & $\begin{array}{c}\mathbf{0 . 4 4} \\
(-10.85)\end{array}$ & $\begin{array}{c}15.84 \\
(15.52)\end{array}$ & $\begin{array}{c}56.19 \\
(56.19)\end{array}$ & $\begin{array}{l}118.92 \\
(118.9)\end{array}$ & $\begin{array}{c}215.67 \\
(215.67)\end{array}$ & $\begin{array}{c}365.06 \\
(365.06)\end{array}$ \\
\hline & $\begin{array}{l}\text { Risk } \\
\text { Prem }\end{array}$ & $\begin{array}{c}0.200 \\
(-)\end{array}$ & $\begin{array}{c}0.142 \\
(0.150)\end{array}$ & $\begin{array}{c}0.099 \\
(0.099)\end{array}$ & $\begin{array}{c}0.087 \\
(0.087)\end{array}$ & $\begin{array}{c}0.082 \\
(0.082)\end{array}$ & $\begin{array}{c}0.080 \\
(0.080)\end{array}$ \\
\hline
\end{tabular}

Table 2: Value and Risk Premium of the R\&D Venture when $\pi$ is Known: The cells in the table show the value and expected excess return of a venture that requires 20 stages to complete for a given $\delta$ and number of completed stages. Bold face values correspond to mothballed projects. The numbers in parentheses give the value and expected return of the venture assuming development must be undertaken, that is, the option to mothball does not exist.

Since $\delta$ has a systematic component, the nonlinearities close to the point where the project is mothballed are also reflected in the risk premium. In Table 2 the bottom two numbers in each cell are the risk premium on the project and, in parentheses, what the risk premium would be if there were no option to abandon or mothball. Comparing these numbers allows us to separately evaluate the contribution of operating leverage and the voluntary exercise decision to the risk premium. 
For high values of $\delta$ and when the project is close to completion, these values are close together even though the risk premium exceeds that earned on the completed project. The risk premium in these cases is attributable to the operating leverage associated with the fixed $R \& D$ expenses, rather than the voluntary nature of those expenses. In the region where the firm is close to exercising the option to mothball, on the other hand, the risk premium on the project is lower than that on the analogous venture with deterministic expenditure. The option to suspend investment truncates the downside of the distribution, lowering both systematic and unsystematic risk. Thus, the "leverage" and the "optionality" embedded in the R\&D venture operate in opposite directions. This is not surprising when one considers an analogy from traditional option pricing theory. An option which is in the money earns a lower risk premium than a forward contract on the same underlying asset when the strike price and forward price are equal. ${ }^{3}$

Figure 1 plots the risk premium (\% per year) against the number of stages completed, $n$, and the value of the cash flow process, $\delta$. The dark line is $\delta^{*}(n)$ (for a given $n$, the highest $\delta$ for which the project is still mothballed). The risk premium is highest when the project is mothballed. The area below the flat region is the region when active development occurs. It is clear from the plot that the risk premium drops dramatically as soon as economic conditions improve to the point that the $R \& D$ is undertaken. It continues to fall towards the risk premium earned by the completed project as circumstances improve and progress continues. The risk premium decreases monotonically over the project's life, consistent with the arguments of Myers and Howe (1997), that the implicit leverage in the project falls as it proceeds, and with the intuition that the project is a compound option on the underlying cash flow process. An important implication of this is that the resolution of technical uncertainty can dramatically affect the risk premium. This is true despite the fact that the technical uncertainty is purely idiosyncratic. Finally, it is evident that this monotonic decrease in the risk premium is not a steady, linear one.

The lower bound the risk premium approaches as the project matures is the risk premium earned by the cash flow process itself. As $\delta(t)$ rises, the risk premium approaches this level at earlier points in the project's life. This is not surprising, since as we saw in Section 4, the value of the R\&D project approaches proportionality in $\delta(t)$, as $\delta(t)$ rises and the abandonment option becomes less important. Finally, note the sensitivity of $\delta^{*}(n)$, the point just before active development begins, to the number of completed stages early on in development. By completing just the first stage it drops from 63 to 38. In contrast, completing the fourteenth stage hardly changes $\delta^{*}(n)$.

It is clear from inspection of Figure 1 that the risk premium approaches, as a lower bound, the premium earned on the completed project. It also appears to flatten out and approach an upper bound in the mothballed region as $\delta$ and $n$ become small. A characterization of this upper bound

\footnotetext{
${ }^{3}$ Consider a security with a payoff $S(T)-K$ at date $T$ where $S(T)$ is the price at $T$ of a stock that pays no dividends between 0 and $T$. The value at $t<T$ of this security is $V(S(t), t)=S(t)-K \exp (-r(T-t))$. By put-call parity $V(t)=C(t)-P(t)$ where $C(t)$ is the price of a call on the stock and $P(t)$ is the price of a put on the stock, both with exercise price $K$ and both European options. The risk premium on the forward contract is equal to $(\alpha-r) \frac{V_{S} S}{V}$ and the risk premium of the call is $(\alpha-r) \frac{C_{S} S}{C}$. Since $V_{S}=1, V(t)=C(t)-P(t)<C(t)$ and since $C_{S}<1$, in the region where $V(t)>0$, the risk premium on the forward is always higher than the risk premium on the call.
} 


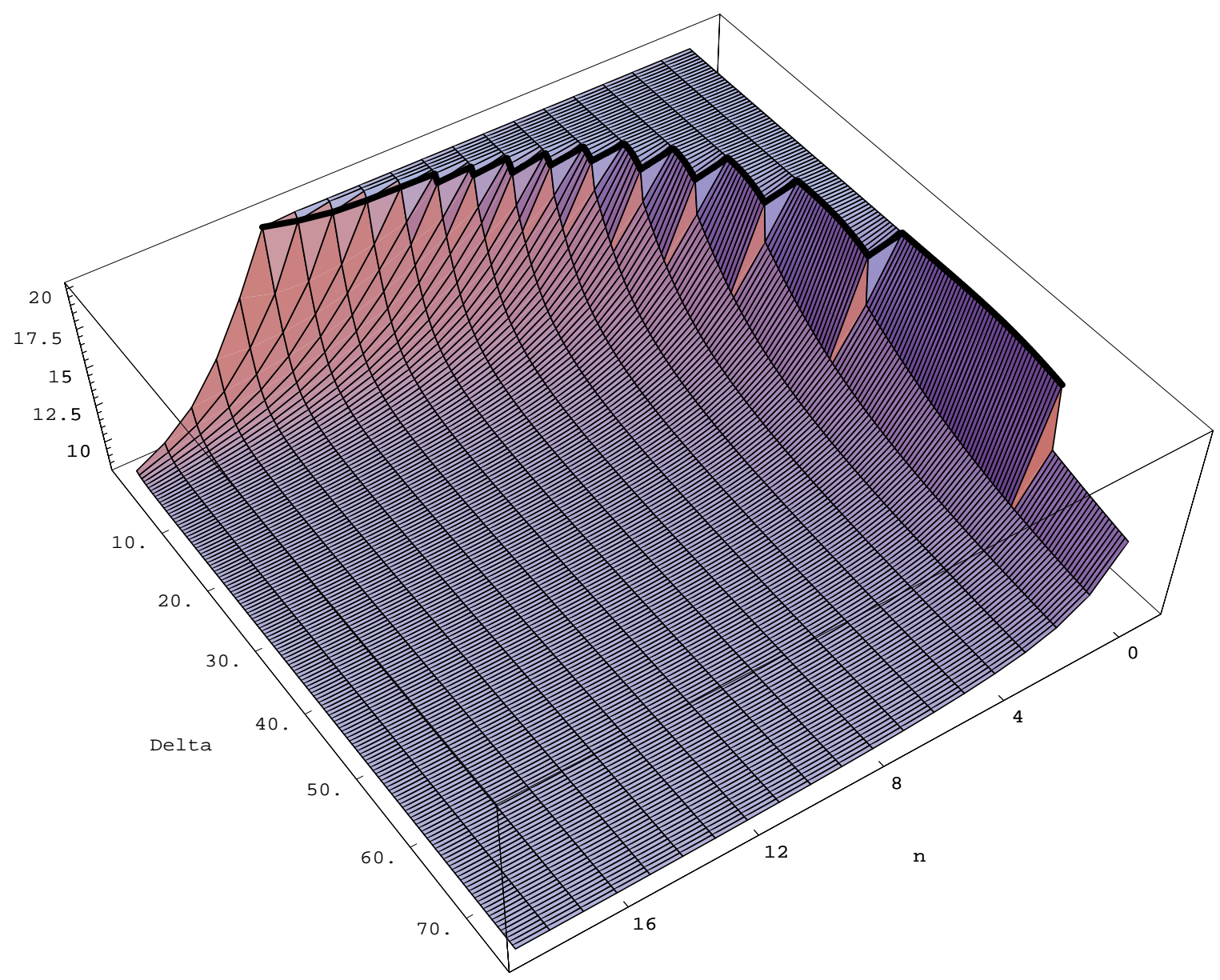

Figure 1: Risk Premium of the R\&D Venture when $\pi$ is known: The risk premium (expressed in \%/annum) of project that requires 20 stages to complete is plotted as a function of $\delta$, (labeled "Delta" in the plot) and $n$, the number of completed stages. The dark line marks, for a given $n$, the highest $\delta$ for which the project is still mothballed, that is, it is $\delta^{*}(n)$. The risk premium is defined to be the difference between the continuously compounded expected return of the project and the riskless rate. 
may be useful in applications, since it provides a very conservative "hurdle rate" for the project.

While we cannot explicitly solve for the value function or the risk premium in our discretetime model, the continuous-time analogue can be used to characterize the point the risk premium approaches in the mothballed region. Suppose the values of $\delta(t), n$ and $m$ are currently such that it is optimal not to invest further in the project. In discrete time the possible values of $\delta(t+1)$ reach across regions of the state space where investment occurs and where mothballing continues. Since the cash flow process cannot jump in continuous time, it possible to solve for the value function "locally" in the region where mothballing occurs. Over this region the risk premium is constant.

Proposition 12 In the continuous-time case, the value function in the region where the project is mothballed has the form $A(n) \delta^{\phi}$, where $\phi$ is a constant, and the risk premium on the RED project is $\phi \lambda$.

Figure 1 shows that the risk premium is relatively constant and at its highest is in the region where it is mothballed. The above result shows that this behavior is attributable to the constant elasticity of the value with respect to the state variable that has systematic risk, $\delta(t)$. We know that the conditional covariance between the $\log$ of $\delta(t)$ and the log of the pricing kernel is constant. Raising $\delta(t)$ to a power greater than one keeps this covariance constant, but increases it. (The expression for $\phi$ derived in the appendix can be shown to be greater than one.) Thus, the analysis provides the bounds we seek. The value of the $R \& D$ venture is most elastic with respect to information about future cash flows, and thus has the most systematic risk and the highest risk premium, when it is mothballed. It is least elastic with regard to this information when the project is complete.

An important feature of the upper bound $\phi \lambda$ is that it is not a function of the probability of success $\pi$. This implies that the analysis can also be applied to the case when $\pi$ is unknown.

It seems quite obvious that as $\delta(t)$ increases and the probability that the firm will exercise all its options to invest approaches unity, the risk premium should approach that earned on the cash flows. It is less obvious that the risk premium is bounded above. The analogy to traditional option pricing theory may be helpful here. In the Black-Scholes model, the risk premium on the call option is equal to the elasticity of the call price with respect to the stock price times the risk premium earned holding the stock. As the stock price approaches zero, this elasticity grows without bound, and so does the risk premium of the option. Because our options are American and do not have an expiration date, the risk premium does not go to infinity, but is in fact bounded above.

\subsection{Unknown Probability of Success}

In the general version of our model, the firm learns about its technical capabilities as it invests in $R \& D$. As we have already argued, although the uncertainty in this process is idiosyncratic, its resolution affects the risk premium.

The importance of uncertainty over technical ability can be gauged by contrasting the values

in Table 3, the value of the project at different stages of development, to those in Table 2, the 


\begin{tabular}{|c||c|c|c|c|c|c||}
\hline \multicolumn{1}{|c||}{} & \multicolumn{7}{c||}{ Number of Completed Stages $(n)$} \\
\multicolumn{1}{|c||}{} & \multicolumn{7}{c||}{ and Failures $(m)$} \\
$\delta$ & 0 & 4 & 8 & 12 & 16 & 20 \\
\hline 5.0 & $\mathbf{0 . 5 7}$ & $\mathbf{0 . 7 2}$ & $\mathbf{4 . 4 8}$ & 20.29 & 48.11 & 91.26 \\
10.0 & 3.25 & $\mathbf{4 . 0 5}$ & 19.93 & 52.12 & 103.13 & 182.53 \\
15.0 & 7.95 & 10.57 & 36.89 & 84.04 & 158.15 & 273.79 \\
\hline
\end{tabular}

Table 3: Value of the $R \& D$ Venture when $\pi$ is Unknown and the number of success equals the number of failures: The cells in the table show the value of a venture that requires 20 stages to complete for a given $\delta$ and number of completed stages, with an equivalent amount of failures (i.e., $m=n$ ). Bold face values correspond to mothballed projects (projects that have an equal number of success and failures are never abandoned for this parameterization).

comparable table when $\pi$ is known. To facilitate this comparison, Table 3 only reports values when $n=m$, since when the number of successes equals the number of failures, $\bar{\pi}(n, n)=0.5$, which is the value for $\pi$ in the case when $\pi$ is known. The extra uncertainty adds considerable value to the project in its early stages. For example, before any research is undertaken, the project is an order of magnitude more valuable when $\pi$ is unknown rather than known. Along with increasing the value of the growth option, the added uncertainty about the probability of success also alters the optimal investment policy. For example, at the outset, when $\delta=10.0$, the R\& $\mathrm{D}$ is mothballed when $\pi$ is known, but is undertaken when it is unknown. In the latter case, undertaking the research allows the firm to obtain a better estimate of $\pi$, and the firm has an option-like claim on this variable. A positive realization of $\pi$ is very valuable, so the firm attempts the research initially in the hopes that the true $\pi$ is greater than $50 \%$. Curiously, although it is initially optimal to invest in R\&D, after four successes and failures it is optimal to mothball, even though the posterior expectation of the true probability of success remains at $50 \%$. Of course, after four success and failures the company has a much more precise estimate of $\pi$. The reduced uncertainty about $\pi$ makes the situation more like the known $\pi$ case, and there it is optimal to mothball with a $50 \%$ chance of success. In general the table shows that as the number of successes and failures increases, the value of the project approaches the value in the known $\pi$ case. ${ }^{4}$

The effect of the uncertainty in $\pi$ is also evident in the plot of the risk premium when the number of successes equals the number of failures. (See Figure 2.) When the number of success and failures is large, the plot looks very similar to Figure 1. However, when there have been very few success and failures, holding constant the posterior probability of success, for high values of $\delta$ the risk premium can increase as the project proceeds. In this case the decreased uncertainty in $\pi$ raises $\delta^{*}$, thus making it more likely that a $\delta$ realization will occur that will mothball the project.

\footnotetext{
${ }^{4}$ The value in the unknown case can actually be lower when $\mathrm{n}$ is large (e.g., $\left.n=16\right)$. This results, presumably, from the asymmetry in the posterior distribution of $\bar{\pi}$ induced by the fact that in the latter stages, $N<<m^{*}(N)$. So, for example, when $n=16$ the absolute most that $\bar{\pi}$ can depart from its current value in the positive direction is $\frac{16+4}{32+4}=55 \%$. On the other hand the negative departure can basically go all the way to $\frac{16}{32+232}=6 \%$, since it takes up to 232 failures to abandon.
} 


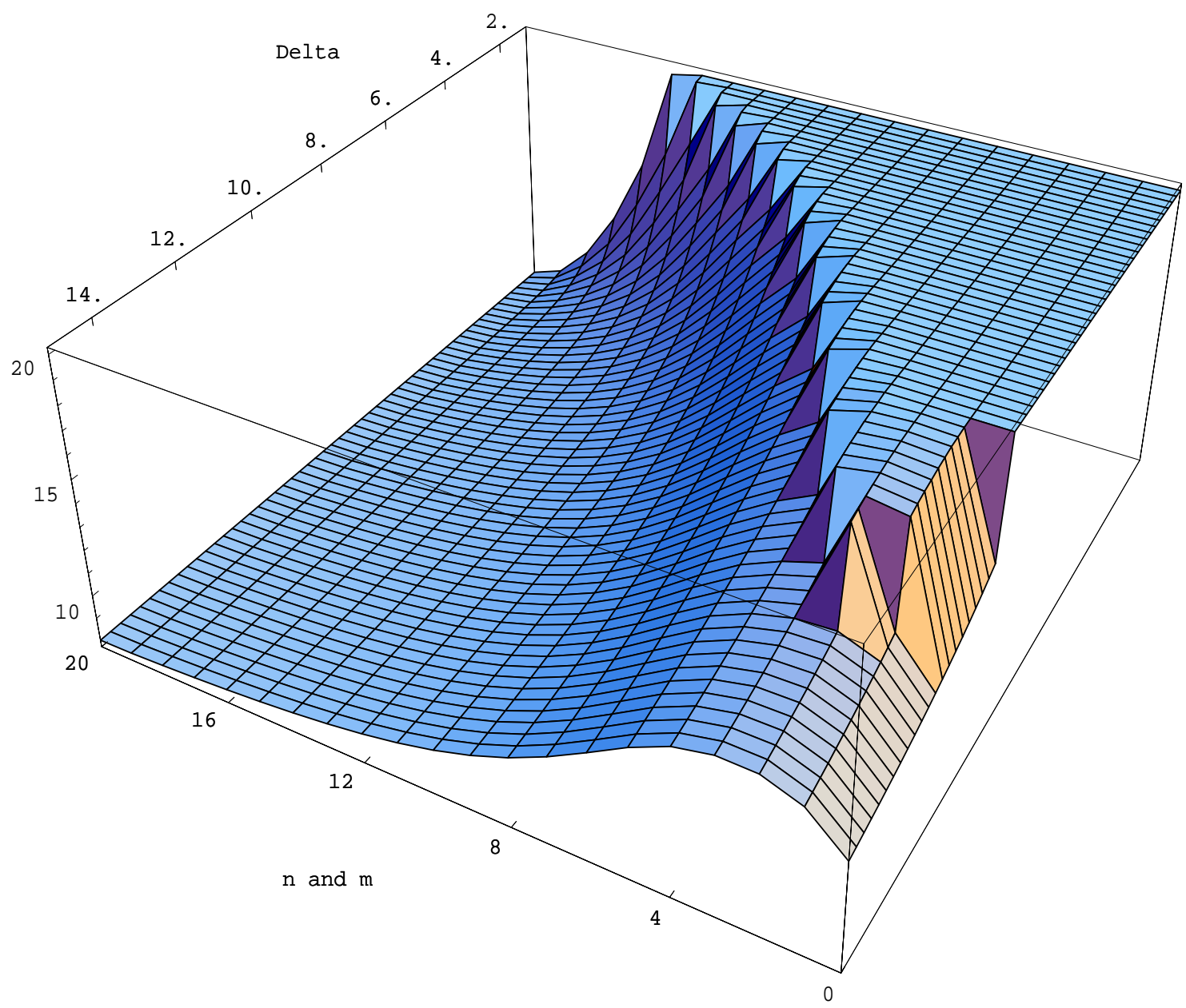

Figure 2: Risk Premium of the R\&D Venture when $\pi$ is Unknown and the number of success equals the number of failures: The risk premium (expressed in \%/annum) of project that requires 20 stages to complete is plotted as a function of $\delta$, (labeled "Delta" in the plot) and the number of completed stages $(n)$ and failures $(m)$. The risk premium is defined to be the difference between the continuously compounded expected return of the project and the riskless rate. 
The early history is so informative that this decreased uncertainty swamps the countervailing effect that the number of stages left to complete is smaller.

\begin{tabular}{|c|c|c|c|c|c|c|c|c|c|c|c|c|c|c|c|c|c|c|c|c|}
\hline \multicolumn{10}{c|}{ Number of Completed Stages or Past Successes $(n)$} \\
0 & 1 & 2 & 3 & 4 & 5 & 6 & 7 & 8 & 9 & 10 & 11 & 12 & 13 & 14 & 15 & 16 & 17 & 18 & 19 \\
\hline 2 & 4 & 6 & 8 & 11 & 14 & 18 & 22 & 28 & 35 & 44 & 55 & 69 & 89 & 118 & 161 & 232 & 373 & 753 & 3430 \\
\hline
\end{tabular}

Table 4: Number of Failures That Trigger Abandonment: The cells in the table show, for a given number of completed stages (or past successes), the number of failures at which it is optimal to abandon the 20 stage venture.

The effect of early learning by doing is even more clearly seen in Table 4, which lists the number of failures that trigger the abandonment decision. Early in the life of an R\&D project technical failure is very costly. Initially, with just two failed attempts and no successes it is optimal to abandon the project. If the true probability of success is $50 \%$, then $25 \%$ of the time the project will be abandoned before even one stage is complete. Later in the life of an $\mathrm{R} \& \mathrm{D}$ project, failure is much less costly. With 19 stages complete, it would take 3430 failures before the abandonment option is exercised. The reason for this does not derive exclusively from the effect of past success on posteriors. It also reflects the fact that the expected cost to completion is lower when only a few stages remain. Nevertheless, these numbers emphasize the important role luck plays in which projects firms ultimately develop. Two identical firms doing R\&D on identical projects can, because of small but early differences the resolution of the technological uncertainty, be led to completely different outcomes.

Because the risk premium is very sensitive around the mothball point, Figure 2 can also be used to assess the differences between investment policies when $\pi$ is known and when it is unknown. The region were the project is mothballed corresponds to the plateau where the risk premium is flat and close to the upper bound calculated in Proposition 12. The critical values that trigger suspension of investment can be deduced from the points at which the plot jumps up to this plateau. Initially, this point occurs at $\delta^{*}(0,0) \approx 9.5$ (see Figure 2 ). However, when $\pi$ is known, this occurs at $\delta^{*}(0) \approx 63$ (see Figure 1). As we have already argued, this difference occurs because when $\pi$ is unknown, one reason to develop is to learning about your technical ability. As this uncertainty is resolved the differences between the optimal investment policies disappear. So, for example, with $n=m=15$, in both cases $\delta^{*}$ is close to 1 .

The ratio of successes to failures also has an important effect on the risk premium. Figure 3 plots the risk premium as a function of the current $\delta$ and the number of past failures at four different stages of development. For example, when $\delta=10$, it is optimal to develop initially and so the risk premium is $15 \%$ per year (Figure 3, upper left panel). With just one failure, the project is mothballed and so the risk premium jumps to the upper bound of $20 \%$. (Areas below the flat parts of the plot are regions in which the project is undertaken and the empty areas in the plots correspond to values for which the venture is abandoned.) On the other hand when 11 stages are 
completed without any failures (Figure 3, upper right panel), for the same value of $\delta$, the risk premium is only $8.75 \%$, close to the lower bound. A failure at this point changes the premium only marginally, to $8.86 \%$. The situation is quite different when the number of past failures is large. If there have been 16 past failures, the risk premium is $16.62 \%$ and it jumps to $19.55 \%$ if one more failure is realized. With 19 stages complete (Figure 3, lower right panel), the project is so far along that unless the number of failures is very large (in excess of 200 - note the scale change on the plot) the number of past failures makes very little difference and the risk premium is within $1 \%$ of the lower bound.

The case with 16 completed stages in Figure 3 (lower left panel) provides a nice illustration of the difference between the decision to mothball and abandon. Recall that the resolution of technological uncertainty is idiosyncratic. Consequently, the reason the decision to mothball affects the risk premium is that the optimal exercise policy depends on the current level of $\delta$. The decision to abandon, however, depends only on the number of past failures, so this option does not command a risk premium. This can be clearly discerned from the plot. When $\delta$ is small, it is possible that with enough failures, the project will be mothballed. Since the mothball option commands a risk premium, this corresponds to the flat part of the plot. As the number of failures increase the risk premium rises dramatically to the upper bound. When $\delta$ is large, it is never optimal to mothball, although for a large enough number of failures, it might be optimal to abandon. However, since the abandonment option does not command a risk premium, the risk premium increases only slightly ${ }^{5}$ as the number of failures approaches $m^{*}(16)=231$.

In Figure 4 we plot expected cash flows over the next 50 years of a project in its initial stage as a function of the current level of $\delta$. The expected cash flows are calculated by averaging the realized cash flow in each period over 1,000,000 simulation paths for each initial value of $\delta$. We generate each simulation path by starting at $n=0, m=0$, and the given $\delta$. We then draw values for the random variables in each period and implement optimal investment policies. From the figure, the life cycle characteristics of the typical project is quite clear. The current (or first) cash flow is either negative or zero, depending on whether it is optimal to develop. After that expected cash flows are negative, reflecting the strictly positive probability that the project will be in development. They also become less negative the more distant they are in time, reflecting the optimal decision to mothball uneconomical projects, and thus cut expected development costs. Since the positive cash flows associated with the completed project are large, in absolute value, compared to the costs, once the minimum five-year development time has passed the expected cash flows quickly become positive. The increases in the expected cash flow eventually stop and they gradually decline as the probability of obsolescence increases geometrically. ${ }^{6}$

\footnotetext{
${ }^{5}$ This small increase results from the option to mothball, since $\delta^{*}(16, m)$ is increasing in $m$, as is evident from the plot.

${ }^{6}$ Although not evident in the plot, there are cases where the expected cash flows increase after 50 years. A few of the very bad projects are mothballed for long periods of time. Recall that once a project is mothballed, it will not be abandoned because no "learning" about technical productivity takes place. The dynamics of the $\delta(t)$ process, however, mean there is always a chance that such a project will eventually come back on line. This will happen precisely when $\delta(t)$ is extremely large, leading to very large, though unlikely, cash flows.
} 

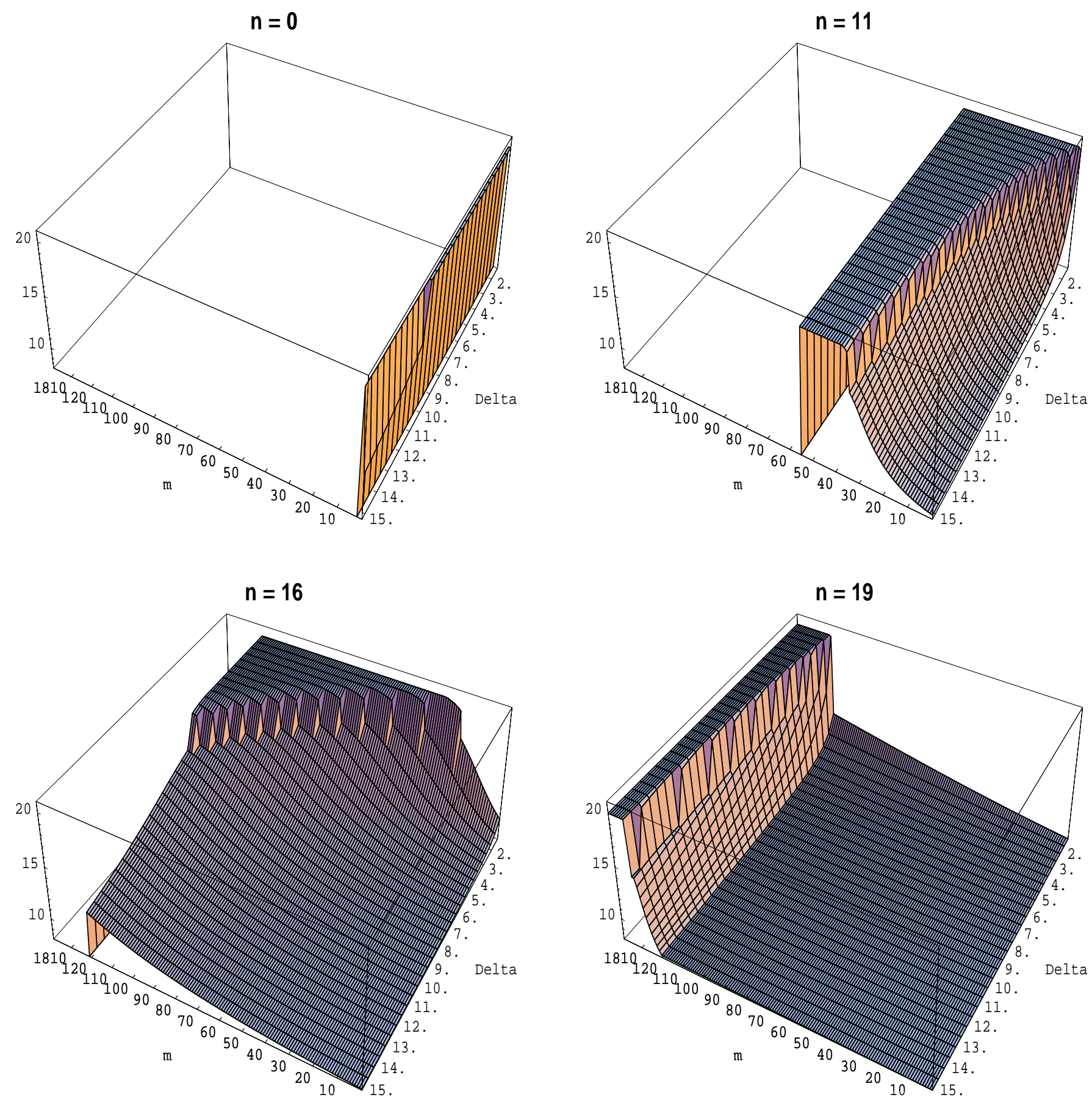

Figure 3: Risk Premium of the R\&D venture when $\pi$ is unknown: For four different stages of completion ( $n=0,11,16$ and 19), the risk premium of a project that requires 20 stages to complete is plotted as a function of $\delta$, (labeled "Delta" in the plot) and $m$, the number of past failures. Note the scale change on the " $\mathbf{m}$ " axis - $m$ is plotted in steps of one from 1 to 120 and then in steps of 169 to 3429 . Blank areas correspond to values for which the venture is abandoned. The risk premium is defined to be the difference between the continuously compounded expected return of the project and the riskless rate. 


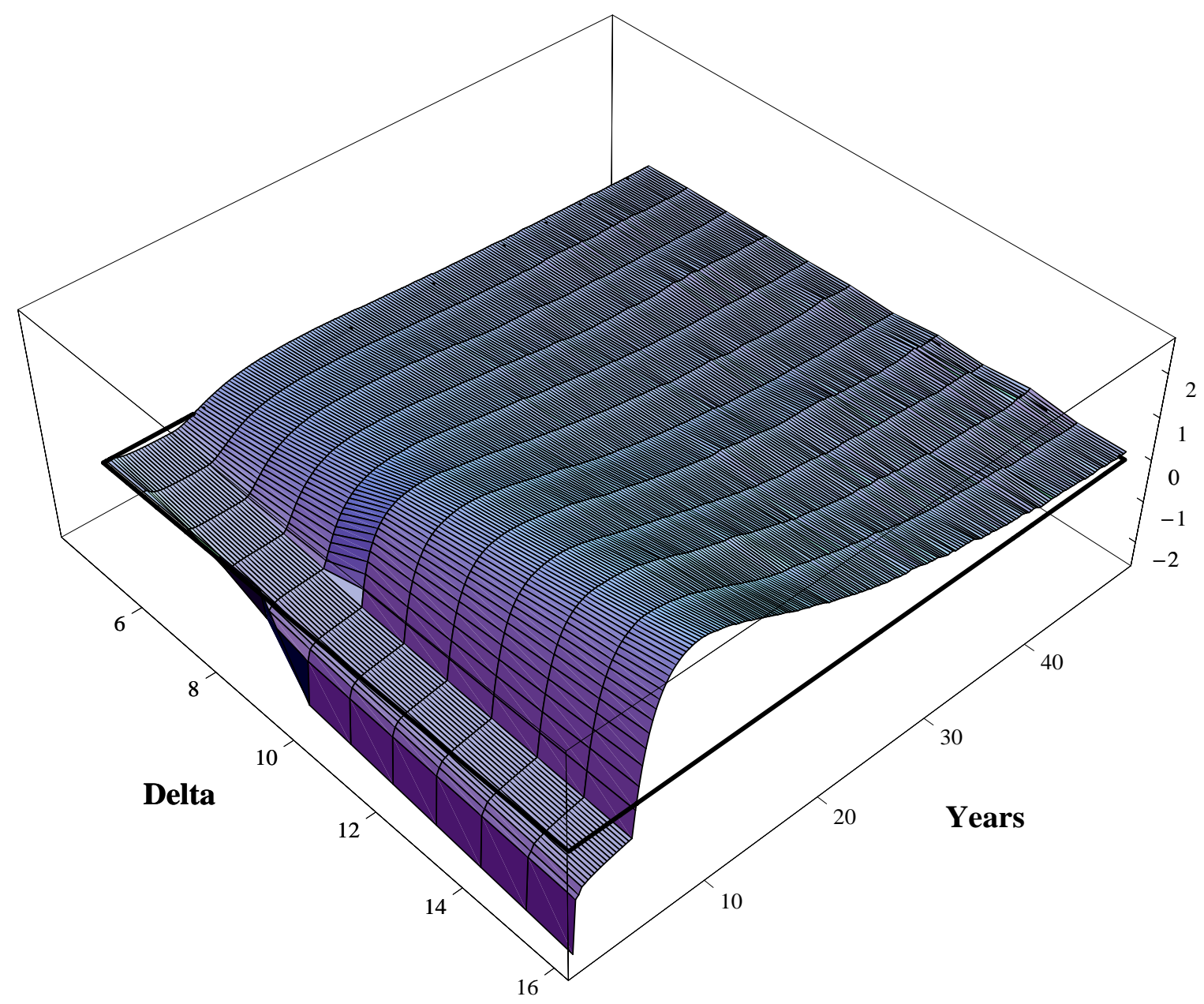

Figure 4: Expected Cash Flow at the Initial Stage of the R\&D Venture when $\pi$ is Unknown: The expected cash flow for a given initial $\delta$ is calculated as follows. 1,000,000 sample paths over 524 periods (131 years) are independently simulated. The expected cash flow is then calculated by averaging the cash flow in each period over all the sample paths. The plot shows this average over the first 200 periods (50 years). The empty black box marks zero on the plot. 


\begin{tabular}{|c|c|c|c|c|c|c|}
\hline \multirow[b]{2}{*}{$\delta$} & & \multicolumn{5}{|c|}{$\begin{array}{l}\text { Number of Completed Stages }(n) \\
\text { and Failures }(m)(n=m)\end{array}$} \\
\hline & & 0 & 5 & 10 & 15 & 20 \\
\hline \multirow{3}{*}{5} & Cost of Cap. & 16.45 & 18.89 & 16.4 & $\bar{~} 13.06$ & 15. \\
\hline & IRR & 19.22 & 23.27 & 25.61 & 53.89 & \\
\hline & Exp. Ret. & 26.97 & 26.97 & 21.61 & 16.39 & 15 . \\
\hline \multirow{3}{*}{7} & Cost of Cap. & 16.24 & 18.7 & 15.41 & 13.09 & 15. \\
\hline & IRR & 19.27 & 23.36 & 28.44 & 62.96 & \\
\hline & Exp. Ret. & 26.97 & 26.97 & 19.25 & 15.94 & 15 . \\
\hline \multirow{3}{*}{9} & Cost & 15.89 & 20.04 & 15.05 & 13.06 & 15. \\
\hline & IRR & 19.17 & 23.05 & 30.95 & 69.27 & \\
\hline & Exp. Ret. & 26.97 & 23.66 & 18.06 & 15.72 & 15 . \\
\hline \multirow{3}{*}{11} & Cost of Cap. & 17.21 & 19.22 & 14.84 & 13.02 & 15. \\
\hline & IRR & 19.17 & 23.67 & 32.86 & 74.09 & \\
\hline & Exp. Ret. & 21.20 & 22.18 & 17.38 & 15.58 & 15 . \\
\hline \multirow{3}{*}{13} & Cost of Cap. & 16.88 & 18.68 & 14.67 & 13.01 & 15. \\
\hline & IRR & 19.8 & 24.18 & 34.47 & 77.76 & \\
\hline & Exp. Ret. & 20.07 & 21.07 & 16.94 & 15.48 & 15. \\
\hline \multirow{3}{*}{15} & Cost of Cap. & 16.59 & 18.14 & 14.62 & 12.94 & 15. \\
\hline & IRR & 20.23 & 24.47 & 35.73 & 80.58 & \\
\hline & Exp. Ret. & 19.3 & 20.18 & 16.64 & 15.41 & 15 . \\
\hline
\end{tabular}

Table 5: IRR and single period (one quarter) expected return of the R\&D Venture when $\pi$ is unknown: The table shows the cost of capital, IRR and expected return over the next quarter of a venture that requires 20 stages to complete at different stages of development when $n=m$. The values are expressed on an annual basis in percent for different values of $\delta$. The IRR (cost of capital) is calculated for each value of $\delta$ by simulating the cash flows over 1,000,000 sample paths and then finding the discount rate that sets the present value of the average cash flows equal to the current net investment (value). Bold face values indicated mothballed projects 
In our model the expected return associated with buying the project at its market value and holding it for one period changes over the project's life. More widely employed capital budgeting procedures typically express the project's profitability and the cost of funds in terms of constant discount rates that apply to all expected cash flows in the future. Accordingly, in Table 5 we use the simulated expected cash flows to calculate the internal rate of return (IRR) and a constant "cost of capital" for the project for a range of values of $\delta$, and at several stages of development. We focus on cases where $n=m$, so that posterior probabilities of success are constant. The IRR is the discount rate that sets the present value of all the project's expected physical cash flows equal to zero. The cost of capital sets the present value of the project's expected cash flows equal to the project's value. (Thus, the cost of capital is always less than the IRR here because the project's value is positive.) The table also reports the project's expected return for comparison. Note that the expected cash flows reflect optimal policies.

From the table we see that the IRR for the project increases with progress towards completion, as one would expect of a measure of the project's attractiveness. The constant cost of capital, however, is non-monotonic, and rises over the early stages of development. This appears to be due to the decline in uncertainty about the value of $\pi$, which, by decreasing the value of the real options embedded in the project, lowers the project's value disproportionately relative to the expected cash flows. Note also that the IRR of the project sometimes lies below its expected return even in the area where it is optimal to make the investment (e.g., in the cell $\delta=13, n=m=0$ ). This is not surprising, as the IRR is a measure of average return earned over the life of the project, while the expected return applies to an investment in the project for the current period.

\subsection{Distribution of Realized Returns}

We now turn to the distribution of realized returns. We first derive this distribution, analytically, for the case with no fixed costs where we have a closed-form solution for the value. We then use numerical methods to derive the distribution in the general case.

We can show analytically for the simplest case that the realized returns are bimodal.

Proposition 13 When there are no fixed RESD costs $(a=0)$ the distribution of the single-period continuously compounded return, conditional on survival, of a project with $n<N$ stages completed and $m<m^{*}(n)$ failures is given by a mixture of two Normal distributions with means $K(n, m)+$ $\log (h(n+1, m))$ and $K(n, m)+\log (h(n, m+1))$, the same standard deviation $\left(\sigma_{\delta}\right)$ and mixture probability $\bar{\pi}(n, m)$ for the former distribution and $1-\bar{\pi}(n, m)$ for the latter, where,

$$
K(n, m) \equiv \mu-\frac{\sigma^{2}}{2}-\log (b+h(n, m))
$$

To understand why the distribution has this form, recall that in this case it is never optimal to mothball the project. Since the return when the project is abandoned is uninteresting, we can 
limit attention to cases in which development is being undertaken. There are two different kinds of uncertainty that affect value. First there is the uncertainty in $\delta(t+1)$, which is normally distributed. Then there is the uncertainty associated with the technical risk. Since a stage is either completed or not, this uncertainty has a discrete distribution. The mixture results from the combination of these two effects.

To examine the realized returns in the general case, we simulate the single period realized return of the project analyzed in the previous section at different levels of $\delta(t)$. Figure 5 are the resulting histograms. What is particularly striking about the figure is how variable realized returns are the plots show that monthly realized returns of $-350 \%$ or $+100 \%$ are quite likely.

We have shown that as $\delta$ increases, values and policies with positive fixed costs converge to the no-fixed-costs case. It is therefore not surprising that when $\delta(t)$ is large the resulting distribution is a mixture of two distributions. Since the probability of success is function of past performance, the mixture probabilities (relative weights) of the two distributions depends on the stage of completion.

The form of the distribution of realized returns changes in a discontinuous way at $\delta=\delta^{*}(0,0)$ (approximately 9.54 for our parameterization). For values of $\delta(t)$ above this the research is undertaken and the form of the distribution mirrors the no-fixed-costs case. When $\delta(t)$ is below this number the distribution is qualitatively different. This corresponds to the case when the project is mothballed. Since no technical uncertainty is resolved for mothballed projects, one might reason that the distribution will no longer be bimodal. In fact, as the case when $\delta(t)=9.539$ in Figure 5 shows, even when the project is mothballed, the distribution of returns results from a mixture of two distributions. One portion of the distribution corresponds to ranges of $\delta(t+1)$ where the project remains mothballed and the other corresponds to the ranges of $\delta(t+1)$ where the project will be undertaken.

Figure 6 contains histograms of realized returns of a project at different stages of development for $\delta=9$. At this level of $\delta$, the project is under active development for $n=m=5$ (upper left panel). By comparing this panel to the lower right panel in Figure 5, the effect of the information revelation associated with the number of completed stages (failures) is evident. In both cases the project is under development, however in the panel in Figure 5 returns are much more diffuse, reflecting the higher level of uncertainty (about $\pi$ ). In both cases a failure at this point will likely cause the project to be mothballed. However, in the case depicted in Figure 6, because of the decreased uncertainty associated with the number of past failures, there is some likelihood of a good enough resolution in $\delta$ that even one more failure can be tolerated without mothballing the project. Similarly, there is some likelihood of a bad enough resolution in $\delta$ that even a project that successfully completes another stage could be mothballed. Thus the two distributions that form the mixture in upper left panel of Figure 6 "overlap."

When the project is even further along, $n=10, m=5$ (lower left panel of Figure 5) or $n=m=10$ (lower right panel of Figure 6), there is no realistic probability that over the next period the project will be mothballed, so the distribution is single peaked. The upper right panel, 

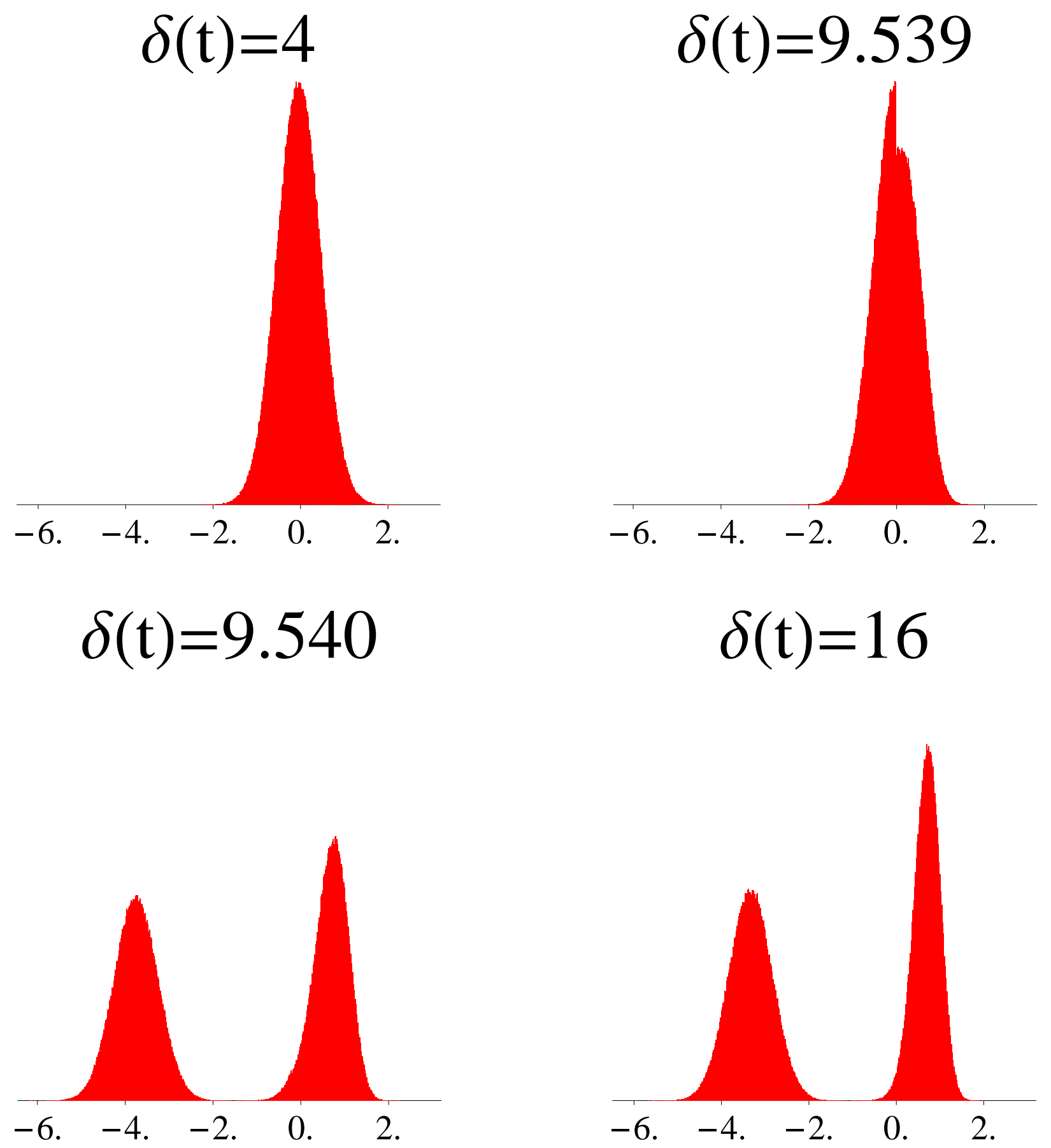

Figure 5: Realized Return of the R\&D venture (conditional on survival) when $\pi$ is unknown: For a project at its initial stage $(n=m=0)$ the single period continuously compounded return of a project is calculated over 1,000,000 repetitions at four different values of $\delta(t)$. The figures show the resulting distribution of returns as a function of the current $\delta(t)$, given that the project survived (i.e., $x(t+1)=1)$. 


\section{$\mathrm{n}=\mathrm{m}=5$}

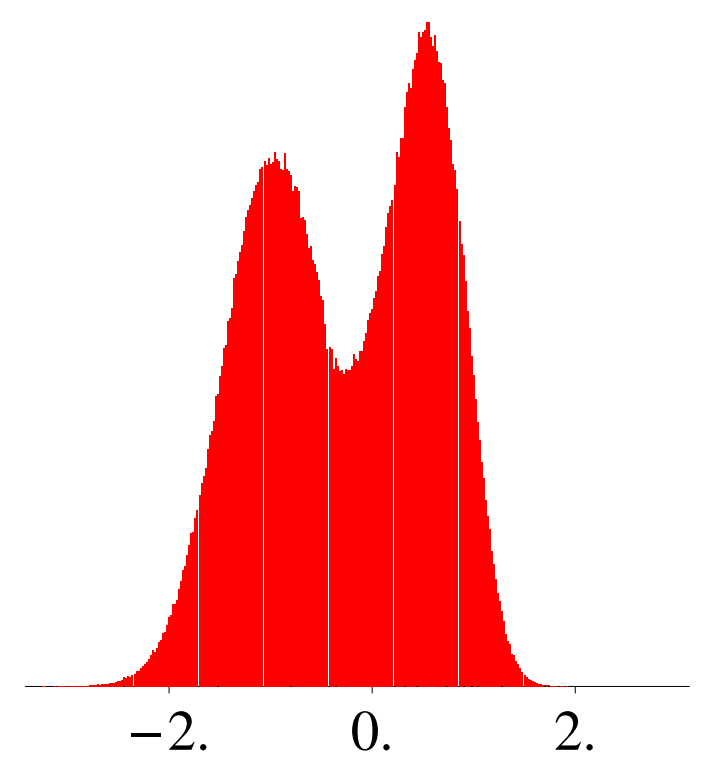

\section{$\mathrm{n}=10 \mathrm{~m}=5$}

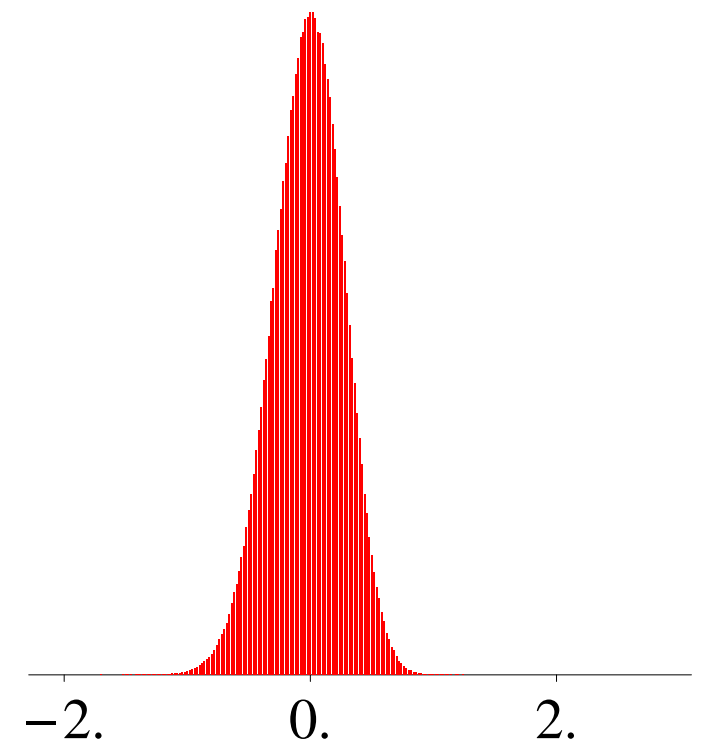

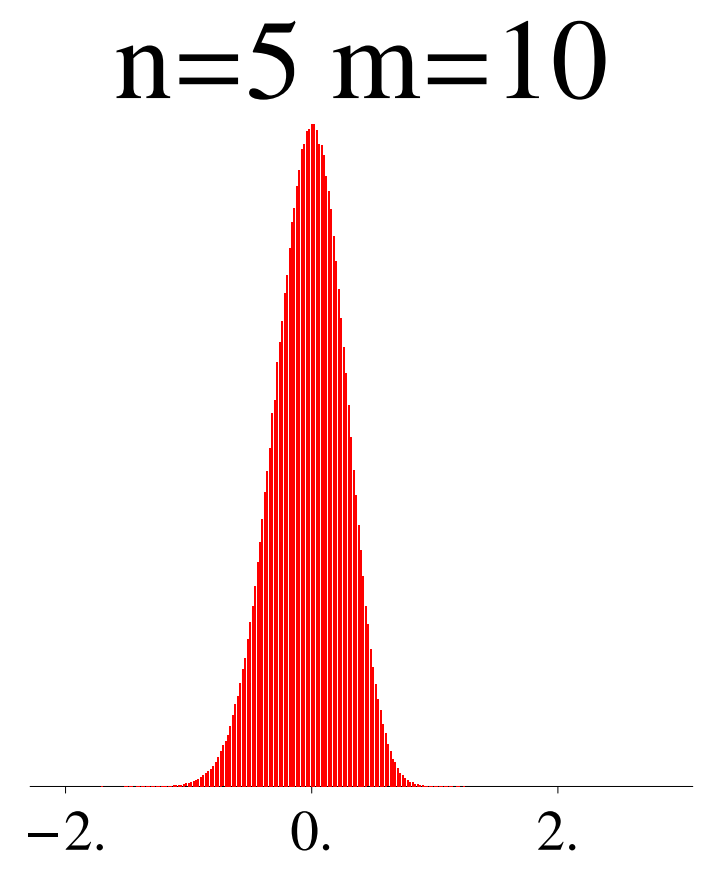

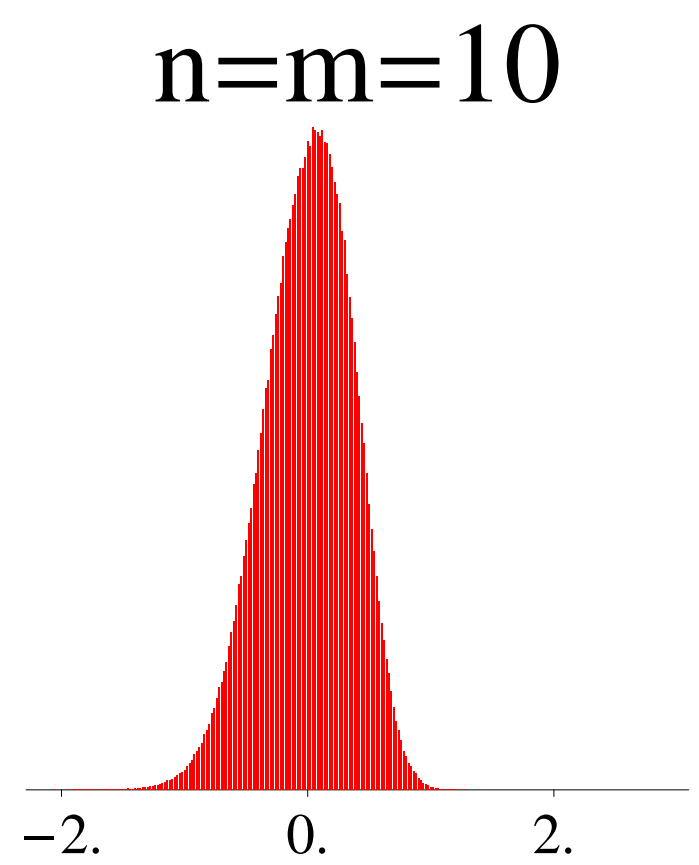

Figure 6: Realized Return of the R\&D venture (conditional on survival) when $\pi$ is unknown and $\delta=9$ : For a project at four different stages in its development the single period continuously compounded return of a project is calculated over 1,000,000 repetitions at four different stages of development. The figures show the resulting distribution of returns as a function of the current $\delta(t)$, given that the project survived (i.e., $x(t+1)=1$ ). 
$n=5, m=10$, shows what happens to the project if it does not progress further. In this case, with five more failures, the optimal policy is to mothball the project. With $\delta=9$, there is no realistic probability that positive resolution in $\delta$ uncertainty in the next period can change this, so again the distribution is singled peaked and reflects the distribution of returns of a mothballed project.

The distribution of realized returns also illustrates nicely the separate roles of systematic and unsystematic risk. The variance of returns when the project is mothballed is clearly considerably smaller than when it is under development and the bimodality is so extreme (e.g., compare the two top panels of Figure 6). Yet the risk premium during the mothball stage is much larger than the development stage. The reason for this disparity is that the extra variance in the realized return of projects under development derives directly from the increase in technological risk. This risk is idiosyncratic, so it does not affect the risk premium.

\section{Conclusion}

$\mathrm{R} \& \mathrm{D}$ and similar investment projects have the property that much of the value of the investment is associated with future cash flows that are contingent on intermediate decisions. The uncertainty these projects involve is of two distinct types. There is purely idiosyncratic risk associated with the resolution of technical uncertainty. There is also risk associated with cash flows after development is complete that will have an important systematic component.

Our analysis highlights the importance of the nature of the information decision makers condition on when they make the intermediate investment decisions to continue, expand, contract, or abandon the project. Whenever that information includes variables, such as forecasts of postdevelopment cash flows, that have a systematic component, this will impart considerable systematic risk to the project, even when the development process itself involves only technical risk. Our results show that the systematic risk, and the required risk premium, of the venture are highest early in its life, and decrease as it approaches completion, despite the idiosyncratic nature of the technical risk. The option-like characteristics of the venture also determine the nature of the dependence of its value on the underlying state variables. At certain times during the project's life, its value is extremely responsive to information about technical progress or potential profitability, and at others much less so. 


\section{Appendix}

\section{A Proofs}

\section{Derivation of the Value Function}

In equation (9), note that the function $V(\cdot)$ appears on both sides, and $V(\cdot)$ is defined as a solution to a functional equation. Below we first show that the functional equation implicit in (9) has a unique solution. Then, we show that this solution indeed provides the value of the $R \& D$ project considered in the paper.

Define $Z \equiv \mathcal{R}_{+} \times \mathcal{N}_{+}$where $\mathcal{N}_{+} \equiv\{0,1,2, \ldots\}$. Let $\mathcal{C}(Z)$ denote the space of continuous functions defined on $Z$ which is a complete and separable metric space. Let $A=\frac{1}{1-\beta}$ and let $\mathcal{D}$ denote a subset of $\mathcal{C}(Z)$ defined by

$$
\mathcal{D} \equiv\left\{f \in \mathcal{C} \mid \sup _{(z, m) \in \mathcal{R}_{+} \times \mathcal{N}_{+}}\left[\frac{|f(z, m)|}{z}\right] \leq A\right\} .
$$

$\mathcal{D}$ is a closed subset of $\mathcal{C}(Z)$ and is, therefore, itself a complete, and separable metric space.

For $n=0,1, \ldots, N-1$, consider a family of mappings $A_{n}: \mathcal{D} \rightarrow \mathcal{C}$ defined recursively by: $\left(A_{n} f\right)(z, m)=\max \left[\left(B_{n} f\right)(z, m),(C f)(z, m)\right]$ where $\left(B_{n} f\right)(z, m)$ equals

$$
\kappa \bar{\pi}(n, m) \int V_{n+1}(z \exp (y), m) n(y) d y+\kappa[1-\bar{\pi}(n, m)] \int f(z \exp (y), m+1) n(y) d y-a-b z,
$$

and

$$
(C f)(z, m)=\kappa \int f(z \exp (y), m) n(y) d y,
$$

$V_{n+1}(\cdot)$ denotes the unique fixed point of $A_{n+1}$ (whose existence is shown below) and where $V_{N}(z, m) \equiv A z$. Moreover, $n(y)$ denotes the normal density with mean $\mu-\lambda-.5 \sigma^{2}$ and variance $\sigma^{2}$ and $\kappa=\pi_{y} \exp (-r)<1$.

Beginning with $n=N-1$, it can be shown recursively for $n=0,1, \ldots, N-1$, that (i) if $f(\cdot) \in \mathcal{D}$, then both $B_{n} f$ and $C f$ are in $\mathcal{D}$. Thus, $A_{n}(\cdot)$ maps $\mathcal{D}$ into $\mathcal{D}$ for all $n=0, \ldots, N-1$, (ii) for all $n, A_{n}$ is monotonic (i.e. $A_{n} f \geq A_{n} g$ whenever $f \geq g$ ), and (iii) with $1_{Z}(z, m) \equiv 1$ for $(z, m) \in Z$ and $\alpha \geq 0$,

$$
A_{n}\left(f+\alpha 1_{Z}\right) \leq \max \left[B_{n} f+\kappa \alpha 1_{Z}, C f+\kappa \alpha 1_{Z}\right]=A_{n} f+\kappa \alpha 1_{Z} .
$$

Then, by Blackwell's fixed point theorem (Duffie (1988), p. 191) $A_{n}$ is a contraction for $0 \leq n \leq$ $N-1$ (since $\kappa<1$ ) and has a unique fixed point. Denote the fixed point of $A_{n}$ by $V_{n}(\delta, m)$. This proves the existence of a unique solution to the functional equation implicit in (9). To make clear the dependence of the value of the $\mathrm{R} \& \mathrm{D}$ project at different stages on $n(t)$, we denote the unique fixed point of $A_{n}, V_{n}(\delta, m)$, also as $V(n, m, \delta)$. 
Define $W[t]$ to be the value of the R\&D project at date $t$. When $x(t)=0, W[t]=0$. Thus, only the case when $x(t)=1$ needs to be analyzed further. We now show that $V(n, m, \delta)$ is indeed the value at date $t$, (when $\delta(t)=\delta, n(t)=n m(t)=m$ and $x(t)=1$ ) of the R\&D project considered in this paper. To see this, consider the problem of valuing an $R \& D$ project that is similar in all respects to the one described in Section 3 except that the project is finitely lived. That is, if the R\&D project has not been finished by time $T$, it becomes worthless after $T$. The value of such an opportunity at any $t$, denoted $W(t, T)$, can be calculated by backward recursion. Evidently, $W(t, T)=0$ for all $t>T$. Also, $W(t, T)=0$ for any $t \leq T$ if $x(t)=0$. Moreover, by backward recursion starting at $T-1$, for any $t \leq T, W(t, T)$ equals $J_{n}(\delta(t), m(t), t, T)$ if $n(t)=n$ and $x(t)=1$, where the functions $J_{n}(\cdot, t, T)$, for $n=0,1, \ldots, N-1$, are defined by $J_{n}(\cdot, t, T)=\max \left[K_{n}(\cdot, t, T), L_{n}(\cdot, t, T)\right]$ with $K_{n}(x, m, t, T)$ given by

$\kappa \bar{\pi}(n, m) \int J_{n+1}(x \exp (y), m, t+1, T) n(y) d y+\kappa[1-\bar{\pi}(n, m)] \int J_{n}(x \exp (y), m+1, t+1, T) n(y) d y-a-b x$

and

$$
L_{n}(x, m, t, T)=\int J_{n}(x \exp (y), m, t+1, T) n(y) d y,
$$

subject to the boundary conditions that $J_{N}(\delta, m, t, T) \equiv A \delta$ for all $t \leq T$ and $J_{n}(\delta, m, T, T) \equiv 0$ for $n \leq N-1$.

We define the value of an infinitely-lived $R \& D$ opportunity to be the limit of the value of finitely-lived opportunities as the life of the opportunity goes to infinity. Accordingly, we set the value of the infinitely-lived R\&D project considered in our model to be $\lim _{T \rightarrow \infty} W(t, T)$.

Since $J_{n}(\cdot, t, T)$ is monotonically increasing in $T$ and $J_{n}(\delta, m, t, T) \leq A \delta$, for all $T \geq 1$, the limit of $J_{n}(\cdot, t, T)$ as $T \rightarrow \infty$ exists. Denote this limit as $J_{n}(\cdot, \infty)$. Using the monotone convergence theorem, it can then be shown that $J_{n}(., \infty)$ is a fixed point of $A_{n}$. Since there is only one fixed point of $A_{n}, n=0,1, \ldots, N-1$, the value of the infinitely-lived R\&D project at any $t$ equals,

$$
\lim _{T \rightarrow \infty} W(t, T)=J_{n(t)}(\delta(t), m(t), t, \infty)=V(n(t), m(t), \delta(t))
$$

when $x(t)=1$.

\section{Proof of Proposition 1}

A single term in (6) can be written:

$$
E_{t}\left\{\frac{z(t+s)}{z(t)} \delta(t+s) x(t+s)\right\}=\delta(t)\left[\pi_{y} \exp (\mu-r-\lambda)\right]^{s}
$$

This follows from (i) writing $z(t+s)$ and $\delta(t+s)$ in terms of $z(t)$ and $\delta(t)$, (ii) combining exponentials, (ii) applying the formula for the mean of a lognormal, and (iv) recognizing that the only non-zero 
covariance between the innovations in $z(t)$ and $\delta(t)$ is contemporaneous and constant. In addition, $x(t+s)$ is independent of $z(t+s)$ and $\delta(t+s)$, and $E_{t}\{x(t+s)\}=\pi_{y}^{s}$. The formula for an infinite geometric series then yields $(7)$.

\section{Proof of Proposition 2}

The value of the firm given it has passed all $N$ stages given by Proposition $1, \delta(t) /(1-\beta)$, is a cum-dividend value, so:

$$
\log E_{t}\left[1+R_{t+1}^{N}\right]=\log \left(\frac{E_{t}\left[\frac{1}{1-\beta} \delta(t+1) x(t+1)\right]}{\delta(t)\left[\frac{1}{1-\beta}-1\right]}\right)
$$

Since $E_{t}[x(t+1)]=\pi_{y}$ and $E_{t}[\delta(t+1)]=\delta(t) e^{\mu}$, this expected return simplifies directly to

$$
\log E_{t}\left[1+R_{t+1}^{N}\right]=\log \left(\frac{\pi_{y} e^{\mu}}{\beta}\right)=r+\lambda
$$

\section{Proof of Proposition 3}

We must show that $V(n, m, \delta)$ is monotone and convex in $\delta$, for all fixed $m$ and $n$. We first demonstrate convexity.

Since $V(n, \cdot)$ is the fixed point of $A_{n}$, and $A_{n}$ is a contraction, it is sufficient to show that for all $n$, if $f(x, m)$ is convex in its first argument for all $m, A_{n} f$ also has this property. ${ }^{7}$ To see this, note that if $V_{n+1}(\cdot)$ and $f(x, m)$ are both convex for all fixed $m$, then $B_{n} f$ also has this property. The same holds for $C$. Finally, the maximum of two convex functions of $\delta$ is itself a convex function of $\delta$. Thus, if $V_{n+1}(\cdot)$ is convex in $\delta$ for fixed $m$, then $A_{n} f$ also inherits this property. This implies

\footnotetext{
${ }^{7}$ To prove that this is sufficient for showing the desired result, note that for an arbitrary $f \in \mathcal{D}$,

$$
\left\|A_{n} f-V_{n}\right\|=\left\|A_{n} f-A_{n} V_{n}\right\| \leq \kappa\left\|f-V_{n}\right\|
$$

because $A_{n}$ is a contraction and $V_{n}(\cdot)$ is its fixed point. Here, we have used $\|f-g\|$ to denote the distance between $f \in \mathcal{C}$ and $g \in \mathcal{C}$. For $m \geq 2$, let $A_{n}^{m} f \equiv A_{n}\left(A_{n}^{m-1} f\right)$. Then, the above equation implies that $\left\|A_{n}^{m} f-V_{n}\right\| \leq$ $\kappa^{m}\left\|f-V_{n}\right\|$ which converges to zero as $m \rightarrow \infty$. Therefore, the function that is the limit of $A_{n}^{m} f$ equals $V_{n}(\cdot)$ for any arbitrary $f \in \mathcal{D}$. Now suppose that for any $f \in \mathcal{D}$ which is convex in $x$, it is the case that $A_{n} f$ also has this
} property. Then, $A^{m} f$ has this property for all $m$. Therefore, for $\lambda \in[0,1]$,

$$
\begin{aligned}
& V_{n}(\lambda x+(1-\lambda) y, m) \\
& \quad=\lim _{m \rightarrow \infty}\left(A_{n}^{m} f\right)(\lambda x+(1-\lambda) y, m) \\
& \quad \leq \lambda \lim _{m \rightarrow \infty}\left(A_{n}^{m} f\right)(x, m)+(1-\lambda) \lim _{m \rightarrow \infty}\left(A_{n}^{m} f\right)(y, m) \\
& \quad=\lambda V_{n}(x, m)+(1-\lambda) V_{n}(y, m) .
\end{aligned}
$$


that if $V_{n+1}(\cdot)$ is convex in $\delta$ for fixed $m, V_{n}(\cdot)$ will be too. To complete the proof note that $V_{N}(\cdot)$, being linear in $\delta$ is also convex in $\delta$.

To prove that $V_{n}(\delta, m)$ is (weakly) increasing in $\delta$ for fixed $m$, let $a>0$. (When $a=0$, this property follows from the linearity of $V_{n}(\cdot)$ in $\delta$.) It can be checked by recursive substitution that for $n=0,1, \ldots, N-1, V_{n}(\delta, m)=\delta G_{n}(x, m)$ where $x=\frac{a}{\delta}$ and $G_{n}: \mathcal{R}_{+} \times \mathcal{N}_{+} \rightarrow \Re_{+}$is the unique fixed point of a mapping $H_{n}$ from $\mathcal{B}$, the set of bounded and continuous functions on $Z$ to $\mathcal{B}$, defined by: $\left(H_{n} f\right)(x, m)=\max \left[\left(R_{n} f\right)(x, m),(S f)(x, m)\right]$ where $\left(R_{n} f\right)(x, m)$ equals

$$
\begin{gathered}
\beta \bar{\pi}(n, m) \int G_{n+1}(x \exp (-y), m) h(y) d y+\beta[1-\bar{\pi}(n, m)] \int f(x \exp (y), m+1) h(y) d y-x-b, \\
(S f)(x, m)=\beta \int f(x \exp (-y), m) h(y) d y,
\end{gathered}
$$

and where $h(y)$ is the normal density with mean $\mu-\lambda+.5 \sigma^{2}$ and variance given by $\sigma^{2}{ }^{8}$ It is now seen that if $G_{n+1}(x, m)$ is decreasing in $x$ and if $f \in \mathcal{B}$ is decreasing in its first argument, then $R_{n} f$ and $S f$ (and consequently $H_{n}$ ) have this property too. This implies that if $G_{n+1}(\cdot)$ is decreasing in $x$ then so is $G_{n}(\cdot)$. Finally, recall that $G_{N}(\cdot)$ is weakly decreasing trivially because it is constant. Thus, $G_{n}(\cdot)$ is decreasing in $x$ for all $n$. This implies that $V_{n}(\delta, m) \equiv \delta G(x, m)=\delta G_{n}\left(\frac{a}{\delta}, m\right)$ is increasing in $\delta$.

The second part of the proposition is the statement that $V(n+1, m, \delta, 1) \geq V(n, m, \delta, 1) \geq$ $V(n, m+1, \delta)$. The proof is similar to the first part. Consider first the monotonicity in $n$ for fixed $m$ and $\delta$. For a fixed $n$, consider $f \in \mathcal{D}$ that satisfy the condition, $f(x, m) \leq V_{n+1}(x, m)$. It can then be shown that $A_{n} f$ also has this property. This implies that $V(\cdot, m, \delta, 1)$ is increasing in $n$ for all $m$ and $\delta$. The details of this argument follow.

Suppose as the induction hypothesis, that $V_{N}(\delta, m) \geq V_{N-1}(x, m) \geq \ldots \geq V_{n+1}(x, m)$. We need to show that this implies $V_{n+1}(x, m) \geq V_{n}(x, m)$. Pick an arbitrary $f \in \mathcal{D}$ such that $f(x, m) \leq$ $V_{n+1}(x, m)$. Recall that $\left(A_{n} f\right)(x, m)=\max \left[\left(B_{n} f\right)(x, m),(C f)(x, m)\right]$. Moreover, $\left(B_{n} f\right)(x, m)$ equals

$$
\begin{aligned}
& \kappa \bar{\pi}(n, m) \int V_{n+1}(x \exp (y), m) n(y) d y+\kappa[1-\bar{\pi}(n, m)] \int f(x \exp (y), m+1) n(y) d y-a-b x \\
& \quad \leq \kappa \bar{\pi}(n, m) \int V_{n+2}(x \exp (y), m) n(y) d y+\kappa[1-\bar{\pi}(n, m)] \int V_{n+1}(x \exp (y), m+1) n(y) d y-a-b x \\
& \quad=\left(B_{n+1} V_{n+1}\right)(x, m)
\end{aligned}
$$

where we have used the induction hypothesis and the fact that $f(\cdot) \leq V_{n+1}(\cdot)$. Similarly, $(C f)(\cdot) \leq$ $\left(C V_{n+1}\right)(\cdot)$. Therefore, $\left(A_{n} f\right)(\cdot) \leq\left(A_{n+1} V_{n+1}\right)(\cdot)=V_{n+1}(\cdot)$. This implies that $V_{n}(\cdot) \leq V_{n+1}(\cdot)$ if the induction hypothesis holds. To start the induction, it follows from direct computations that $V_{N-1}(\cdot) \leq V_{N}(\cdot)$.

Similarly, it can be shown that if $V_{n+1}(\delta, m)$ is decreasing in $m$ for all $\delta$ and if $f \in \mathcal{D}$ also satisfies this property and the property that $f(x, m) \leq V_{n+1}(x, m)$, then $B_{n} f$ and $C f$ (and consequently

\footnotetext{
${ }^{8}$ The existence of a unique fixed point for $H_{n}$ follows from the arguments presented above for $V_{n}(\cdot)$.
} 
$\left.A_{n} f\right)$ must also be decreasing in $m$ for any $\delta$. This implies that $V(n, m, \delta, 1)$ is decreasing in $m$ for all $n$ and $\delta$ since $V(N, m, \delta, 1)$ does not depend on $m$.

Finally, we need to prove the last part of the proposition, that $\lim _{\delta \rightarrow 0} V(n, m, \delta)=0$. This follows directly from the facts that $0 \leq V(n, m, \delta) \leq V(N, m, \delta)$ and that $V(N, m, \delta) \rightarrow 0$ as $\delta \rightarrow 0$.

\section{Proof of Proposition 4}

First we must establish that if (13) holds then (14) follows. Substitution from (2) and (5), combining exponents, and applying the formula for the mean of a lognormal variable gives:

$$
E_{t}\left\{\frac{z(t+1)}{z(t)} \delta(t+1) x(t+1)\right\}=\exp (\mu-r-\lambda)
$$

Then substituting the conjectured form for the value function, (13), into the definition of $V_{e},(10)$, and recognizing that $\delta(t+1)$ is independent of $x(t+1)$, gives (14). The result now follows by simply substituting the conjectured forms for $V$ and $V_{e}$ into (12), with $a=0$ and recognizing that (15) holds.

\section{Proof of Proposition 5}

The proof of the first part of the proposition follows immediately from the fact that the second argument of the $\max [\cdot, \cdot]$ operator in (16) is the discounted value of the left-hand side of the equation (if the value is finite, $\beta<1$ ). The function $h(n, m)$ cannot equal the discounted value of itself, $\beta h(n, m)$, unless both are zero.

That $h(N, m)=\frac{1}{1-\beta}$ is a direct consequence of Proposition 1 and Proposition 4.

The finiteness of $m^{*}(\cdot)$ follows from the fact when $b>0$, there exists a finite $m$ such that the first argument of the $\max [\cdot, \cdot]$ operator in the right-hand side of (16) is strictly less than zero. We establish this by induction on $n$. Take $n=N-1$. We established above that $h(N, m)$ does not depend on $m$. Clearly, $\lim _{m \rightarrow \infty} \bar{\pi}(N-1, m)=0$. If the first argument in (16 never became negative, then the $\max [\cdot, \cdot]$ operator could be dropped and we would have

$$
h(n, m)-\bar{\pi}(N-1, m) \frac{\beta}{1-\beta}-(1-\bar{\pi}(n, m)) \beta h(N-1, m+1)+b=0 .
$$

Taking the limit as $m$ goes to infinity provides,

$$
\lim _{m \rightarrow \infty} h(N-1, m)-\beta h(N-1, m+1)+b=0
$$

which implies that for large enough $m, h(N-1, m)$ is increasing in $m$. This contradicts Proposition 
3 and so establishes the finiteness of $m^{*}(N-1)$. Now consider $n \leq N-2$. Suppose as the induction hypothesis that $m^{*}(n+1)$ is finite. Using similar arguments we have that if, for a given $n$, the first argument of the $\max [\cdot, \cdot]$ operator did not eventually become negative, then

$$
\lim _{m \rightarrow \infty} h(n, m)-\beta h(n, m+1)+b=0
$$

which contradicts Proposition 3 for the same reason

\section{Proof of Proposition 6}

Note that the definition of $m^{*}$ is just a restatement of (19). Thus the case when $n<N$ and $m>m^{*}(n)$ is trivially true. So is the case when $n=N$. We will show that the remaining functional form satisfies equation (15) above.

First note that the ratio of Pochhammers in this functional form

derives from the following fact:

$$
\frac{\left(m+\alpha_{2}\right)_{i}}{\left(m+n+\alpha_{1}+\alpha_{2}\right)_{i}}=\prod_{j=1}^{i}(1-\bar{\pi}(n, m+j-1))
$$

Using (38) and the definition of $\bar{\pi}$ (equation (12)), the functional form for the last case can be written as

$$
\begin{aligned}
h(n, m+1) & =\sum_{i=0}^{m^{*}(n)-m-1}[\beta \bar{\pi}(n, m+i+1) h(n+1, m+i+1)-b] \beta^{i} \prod_{j=1}^{i}(1-\bar{\pi}(n, m+j)) \\
& =\sum_{i=1}^{m^{*}(n)-m}[\beta \bar{\pi}(n, m+i) h(n+1, m+i)-b] \beta^{i-1} \prod_{j=2}^{i}(1-\bar{\pi}(n, m+j-1)) \\
& =\frac{\sum_{i=1}^{m^{*}(n)-m}[\beta \bar{\pi}(n, m+i) h(n+1, m+i)-b] \beta^{i} \prod_{j=1}^{i}(1-\bar{\pi}(n, m+j-1))}{\beta(1-\bar{\pi}(n, m))} \\
& =\left[\left(\sum_{i=0}^{m^{*}(n)-m}[\beta \bar{\pi}(n, m+i) h(n+1, m+i)-b] \beta^{i} \prod_{j=1}^{i}(1-\bar{\pi}(n, m+j-1))\right)\right. \\
& =\frac{h(n, m)-\beta \bar{\pi}(n, m) h(n+1, m)+b}{\beta(1-\bar{\pi}(n, m))} .
\end{aligned}
$$


Solving for $h(n, m)$ provides

$$
h(n, m)=\beta(1-\bar{\pi}(n, m)) h(n, m+1)+\beta \bar{\pi}(n, m) h(n+1, m)-b
$$

which is $(15)$ when $m \leq m^{*}(n)$.

\section{Proof of Proposition 7}

If $g(0)<0$, it is suboptimal to start the $\mathrm{R} \& \mathrm{D}$. Since the $\mathrm{R} \& \mathrm{D}$ is never started, its value is always zero.

It is straightforward to verify that the following function satisfies (23):

$$
g(N-n)=\gamma^{N-n} \frac{1}{1-\beta}-b \theta \sum_{i=0}^{N-n-1} \gamma^{i}
$$

The expressions for $g(n)$ and $g(0)$ then follow from rewriting the above using the formula for a

finite geometric series. Finally, note that the quantity $\left[\frac{1}{1-\beta}+\frac{b \theta}{1-\gamma}\right]$ is a positive constant, since $\beta$, $\gamma$ and $\theta$ are all positive fractions. So,

$$
g^{\prime}(n)=-\gamma^{(N-n)}\left[\frac{1}{1-\beta}+\frac{b \theta}{1-\gamma}\right] \log \gamma>0
$$

and

$$
g^{\prime \prime}(n)=\gamma^{(N-n)}(\log \gamma)^{2}\left[\frac{1}{1-\beta}+\frac{b \theta}{1-\gamma}\right]>0
$$

\section{Proof of Proposition 8}

It can be verified by direct substitution in the functional equations defining $G_{n}(\cdot)$ in the proof of Proposition 3 above that

$$
\lim _{\delta \rightarrow \infty} \frac{V(n, m, \delta, 1)}{\delta}=G_{n}(0, m)=h(n, m)
$$

where $h(n, m)$ is defined by the recursion in (15). This says that as $\delta \rightarrow \infty$, the effect of fixed cost $a$ becomes negligible on the value of the project and it converges to the value in the case with $a=0$.

\section{Proof of Proposition 9}


The second part of the proposition is just a restatement of Proposition 5. From Proposition 8 we know that as $\delta \rightarrow \infty$, the value of the project converges to the value in the proportional case. But with $b=0$, this value will be positive. This will, because $\delta(t)$ is a geometric random walk, happen with positive probability. Since there is no cost associated with waiting while the project is mothballed, it cannot be optimal to abandon the project.

\section{Proof of Proposition 10}

We first establish that for $m^{*}(n)$ defined in Proposition 6 when $m>m^{*}(n)$ the project is abandoned, that is, $V(m, n, \delta)=0$. When $a=0$, we know that $V(m, n, \delta)=\delta h(n, m)$. From Proposition 5 we know there is always an $m$ sufficiently large that the project is abandoned, so $\lim _{m \rightarrow \infty} h(n, m)=0$ for $n \leq N-1$. The result then follows from the fact that when $a>0$, $0 \leq V(n, m, \delta) \leq h(m, n) \delta$.

Next we show that if $m \leq m^{*}(n)$, the project is never abandoned. First note that for the no fixed cost case, if $m \leq m^{*}(n)$ then $V_{n}(n, m, \delta)>0$, where $V_{n}$ denotes the value when $a=0$. Using this result and Proposition 8, we have,

$$
\lim _{\delta \rightarrow \infty} V(n, m, \delta)=V_{n}(n, m, \delta)>0
$$

Thus, there must always exist a $\delta$ large enough so that for any $m \leq m^{*}(n), V(n, m, \delta)>0$. It can never be optimal to abandon a project for which an attainable state exists in which the value of the project is positive.

We next establish some properties of the derivative of $V_{n}(\cdot)$ with respect to $\delta$. Since $V_{n}(\cdot)$ is convex in $\delta$, it is differentiable almost everywhere. Recall from the definition of $V_{n}(\cdot)$ that it is the maximum of two functions, $B_{n} V_{n}$ and $C V_{n}$, which are convex and (continuously) differentiable in $\delta$. Thus, except when these two functions are equal, $V_{n}(\cdot)$ is also differentiable in $\delta$. It may not be differentiable when $B_{n} V_{n}(\cdot)$ and $C V_{n}(\cdot)$ are equal. In the derivations below, prime denotes the derivative of a function with respect to $\delta$ if it is differentiable or its right-derivative if it is not. The right (and left)-derivatives of $V_{n}(\cdot)$ with respect to $\delta$ exist since $V_{n}(\cdot)$ is convex.

Now we derive an expression for $V_{n}^{\prime}(\cdot)$. Define

$$
\mathcal{A}^{o}(m, n) \equiv\left\{\delta \in \Re_{+} \mid\left(B_{n} V_{n}\right)(\delta, m)>\left(C V_{n}\right)(\delta, m)\right\},
$$

let

$$
\mathcal{A}^{o o}(m, n) \equiv\left\{\delta \in \Re+\mid\left(B_{n} V_{n}\right)(\delta, m)<\left(C V_{n}\right)(\delta, m)\right\}
$$

and, finally,

$$
\tilde{\mathcal{A}} \equiv\left\{\delta \in \Re_{+} \mid\left(B_{n} V_{n}\right)(\delta, m)=\left(C V_{n}\right)(\delta, m)\right\} .
$$


Then, for $\delta \in \mathcal{A}^{o}(m, n)$,

$$
\begin{aligned}
V_{n}^{\prime}(\delta, m) & =\kappa \bar{\pi}(n, m) \int V_{n+1}\left(e^{z}, m\right) g_{\delta}(z ; \delta) d z+\kappa(1-\pi(n, m)) \int V_{n}\left(e^{z}, m+1\right) g_{\delta}(z ; \delta) d z-b \\
& =\beta \bar{\pi}(n, m) \int V_{n+1}^{\prime}(\delta \exp (y), m) h(y) d y+\beta(1-\pi(n, m)) \int V_{n}^{\prime}(\delta \exp (y), m+1) h(y) d y-b
\end{aligned}
$$

where $g(z ; \delta)$ denotes the normal density with mean $\left(\mu-\lambda-.5 \sigma^{2}\right)+\ln (\delta)$ and variance $\sigma^{2}, g_{\delta}(\cdot)$ denotes its derivative with respect to $\delta$, and where $h(\cdot)$ has already been defined as the normal density with mean $\left(\mu-\lambda+.5 \sigma^{2}\right)$ and variance $\sigma^{2}$.

For $\delta \in \mathcal{A}^{o o}(m, n)$,

$$
V_{n}^{\prime}(\delta, m)=\kappa \int V_{n}\left(e^{z}, m\right) g_{\delta}(z ; \delta) d z=\beta \int V_{n}^{\prime}(\delta \exp (y), m) h(y) d y
$$

For $\delta \in \tilde{A}, V_{n}^{\prime}(\delta, m)$ equals

$$
\begin{gathered}
\max \left[\beta \bar{\pi}(n, m) \int V_{n+1}^{\prime}(\delta \exp (y), m) h(y) d y+\beta[1-\pi(n, m)] \int V_{n}^{\prime}(\delta \exp (y), m+1) h(y) d y-b\right. \\
\left.\beta \int V_{n}^{\prime}(\delta \exp (y), m) n(y) d y\right]
\end{gathered}
$$

The above expressions for $V_{n}^{\prime}(\cdot)$ can be deduced from the expression for $V_{n}(\cdot)$. The interchanging of integration and differentiation that the above expressions involve can be justified formally. We omit the details. Similar expressions obtain for the derivatives of the finite-horizon approximations to $V_{n}(\cdot)$.

The next two lemmas prove results on the slope of the components of the value function.

Lemma $1 V_{n}^{\prime}(\delta, m) \leq A$

Proof: We consider the case when $a>0$. (The result follows from direct computations when $a=0$.) Suppose $\delta \in \mathcal{A}^{o}(n, m)$, let $x=\frac{a}{\delta}$. Then, the first expression for $V_{n}^{\prime}(\cdot)$ given in (48) above, and explicit differentiation of $g(z ; \delta)$ with respect to $\delta$, imply that

$$
\begin{aligned}
V_{n}^{\prime}(\delta, m)= & \frac{\kappa \pi(n, m)}{\sigma^{2} \delta} \int V_{n+1}\left(e^{z}, m\right)[z-\ln \delta-\hat{\mu}] g(z ; \delta) d z+ \\
& \frac{\kappa[1-\pi(n, m)]}{\sigma^{2} \delta} \int V_{n}\left(e^{z}, m+1\right)[z-\ln \delta-\hat{\mu}] g(z ; \delta) d z-b \\
= & \frac{\pi(n, m) \beta}{\sigma^{2}} \int G_{n+1}(x \exp (-y), m)[y-\hat{\mu}] h(y) d y+ \\
& \frac{[1-\pi(n, m)] \beta}{\sigma^{2}} \int G_{n}(x \exp (-y), m+1)[y-\hat{\mu}] h(y) d y-b
\end{aligned}
$$

where $\hat{\mu}=\mu-\lambda-.5 \sigma^{2}$ and where $G_{n}(\cdot), n=0 \ldots, N$, have been defined in the proof of Proposi- 
tion 3. Note that $G_{n}(\delta, m) \leq A$. Therefore, the last expression in the above set of equations is no greater than

$$
\frac{\pi(n, m) \beta A}{\sigma^{2}} \int[y-\hat{\mu}] h(y) d y+\frac{[1-\pi(n, m)] \beta A}{\sigma^{2}} \int[y-\hat{\mu}] h(y) d y=\beta A \leq A
$$

because $h(\cdot)$ is the normal density with mean given by $\hat{\mu}+\sigma^{2}$.

The same conclusion can be established for $\delta \in \mathcal{A}^{o o}(n, m)$. Together these two conclusion imply that the same holds for $\delta \in \tilde{\mathcal{A}}(n, m)$.

Lemma $2\left(B_{n} V_{n}\right)^{\prime}(\delta, m) \geq\left(C V_{n}\right)^{\prime}(\delta, m)$

Proof: This result is proved by first proving an analogous result for $J_{n}(\delta, m, 0, T)$, the finitehorizon approximations for $V_{n}(\delta, m)$. Recall that $J_{n}(\delta, m, 0, T)=\max \left[K_{n}(\delta, m, 0, T), L_{n}(\delta, m, 0, T)\right]$ where $K_{n}(\cdot)$ and $L_{n}(\cdot)$ are defined in the derivation of value function above. Note for future reference that since the valuation problem considered here is time-homogeneous, $K_{n}(\delta, m, 0, T) \equiv$ $K_{n}(\delta, m, 1, T+1)$. Similarly, for $L_{n}(\cdot)$. We show that $K_{n}^{\prime}(\delta, m, 0, T) \geq L_{n}^{\prime}(\delta, m, 0, T)$ for all $T \geq 0$. The proof is by induction on $T$. Suppose that $K_{n}^{\prime}(\cdot, 0, T+1) \geq L_{n}^{\prime}(\cdot, 0, T+1)$ for some $T \geq 0$. This means that

$$
\begin{aligned}
K_{n}^{\prime}(\delta, & m, 0, T+1) \\
= & \beta \pi(n, m) \int J_{n+1}^{\prime}(\delta \exp (y), m, 0, T) h(y) d y \\
& +\beta[1-\pi(n, m)] \int J_{n}^{\prime}(\delta \exp (y), m+1,0, T) h(y) d y-b \\
\geq & \beta \int J_{n}^{\prime}(\delta \exp (y), m, 0, T) h(y) d y \\
= & L_{n}^{\prime}(\delta, m, 0, T+1)
\end{aligned}
$$

for $(\delta, m) \in Z$. It can be shown that this implies that

$$
K_{n}^{\prime}(\delta, m, 0, T+1) \geq J_{n}^{\prime}(\delta, m, 0, T+1) \geq L_{n}^{\prime}(\delta, m, 0, T+1)=\beta \int J_{n}^{\prime}(\delta \exp (y), m, 0, T) h(y) d y
$$

We show that this in turn implies that $K_{n}^{\prime}(\cdot, 0, T+2) \geq L_{n}^{\prime}(\cdot, 0, T+2)$. Using the definition of $K_{n}^{\prime}(\delta, m, 0, T+2)$,

$$
\begin{aligned}
& K_{n}^{\prime}(\delta, m, 0, T+2) \\
& =\quad \beta \pi(n, m) \int J_{n+1}^{\prime}(\delta \exp (y), m, 0, T+1) h(y) d y \\
& \quad+\beta[1-\pi(n, m)] \int J_{n}^{\prime}(\delta \exp (y), m, 0, T+1) h(y) d y-b
\end{aligned}
$$




$$
\begin{aligned}
\geq & \beta^{2} \pi(n, m) \iint J_{n+1}^{\prime}(\delta \exp (y+z), m, 0, T) h(z) h(y) d z d y \\
& +\beta^{2}[1-\pi(n, m)] \iint J_{n}^{\prime}(\delta \exp (y+z), m+1,0, T) h(z) h(y) d z d y-b \\
= & \beta \int K_{n}^{\prime}(\delta \exp (y), m, 0, T+1) h(y) d y \\
\geq & \beta \int J_{n}^{\prime}(\delta \exp (y), m, 0, T+1) d y \\
= & L_{n}^{\prime}(\delta, m, 0, T+2),
\end{aligned}
$$

where the inequalities make use of (52) and the equalities make use of definitions. To conclude the induction, it can be verified by direct computations that the induction hypothesis holds for $T=0$.

Recall now that $\lim _{T \rightarrow \infty} K_{n}(\delta, m, 0, T)=\left(B_{n} V_{n}\right)(\delta, m)$ for $n \leq N-1$ and $\lim _{T \rightarrow \infty} L_{n}(\delta, m, 0, T)=$ $C V_{n}(\delta, m)$. Moreover, $K_{n}(\cdot, T), L_{n}(\cdot, T), B_{n} V_{n}(\cdot)$ and $C V_{n}(\cdot)$ are all differentiable and convex. As a result, the derivatives of $L_{n}(\cdot, T)$ converges to the derivative of $B_{n} V_{n}(\cdot)$ and the derivative of $K_{n}(\cdot, T)$ converges to the derivative of $C V_{n}(\cdot)$ by Theorem 25.7 of Rockafellar (1970). Therefore, it must be the case that $\left(B_{n} V_{n}\right)^{\prime}(\delta, m) \geq\left(C V_{n}\right)^{\prime}(\delta, m)$ for $n \leq N-1$.

Using these two lemmas we can now prove the results. First, we show it is optimal to invest whenever $\delta(t)$ is greater than some $\delta^{*}(n, m)$.

Suppose $a>0$. Consider $(m, n)$ such that the value of the R\&D project is strictly positive when $a=0$. (That is, consider $(n, m)$, such that $h(n, m)$ defined in (18) is strictly positive.) Note that (i) for any $n \leq N-1, B_{n} V_{n}(0, m)<0$ and $C V_{n}(0, m)=0$ and (ii) for $n \leq N-1$,

$$
\lim _{\delta \rightarrow \infty} \frac{B_{n} V_{n}(\delta, m)}{\delta}=h(m, n)>\lim _{\delta \rightarrow \infty} \frac{\left(C V_{n}\right)(\delta, m)}{\delta}=\beta h(n, m) .
$$

That is, for fixed $(m, n)$, the function $B_{n} V_{n}(\delta, m)$ starts below $\left(C V_{n}\right)(\delta, m)$ and ends up strictly higher. Moreover, Lemma 2 shows that the derivative of $\left(B_{n} V_{n}\right)(\cdot)$ (with respect to $\delta$ ) is always greater than or equal to that of $\left(C V_{n}\right)(\cdot)$. That means that the functions, $B_{n} V_{n}(\delta, m)$ and $C V_{n}(\delta, m)$ for fixed $(m, n)$ only cross once. Denoting the point of intersection of these two functions as $\delta^{*}(n, m)$, we see that the optimal $\mathrm{R} \& \mathrm{D}$ policy for a given $(\delta, n, m)$ is to continue with the $\operatorname{R} \& \mathrm{D}$ if $\delta \geq \delta^{*}(n, m)$ and to mothball otherwise. If $\delta$ is so low that $V_{n}(\delta, m)=0$, then the policy is to mothball the project forever, that is to abandon the project.

It remains to show that $\delta^{*}(n, m)$ increases as the fixed cost increases.

For a given $a>0$, and for fixed $(n, m), \delta^{*}(n, m ; a)$ is the unique solution to the equation: $\left(B_{n} V_{n}\right)\left(\delta^{*}, m ; a\right)=\left(C V_{n}\right)\left(\delta^{*}, m ; a\right)$. This equation can also written as $\delta^{*}(m, n)=\frac{a}{x^{*}(m, n)}$ where $x^{*}(m, n)$ is the unique solution to the equation: $\left(R_{n} G_{n}\right)\left(x^{*}, m\right)=\left(S G_{n}\right)\left(x^{*}, m\right)$. Neither $R_{n} G_{n}$ nor $S G_{n}$ depends on $a$. Therefore, $x^{*}(m, n)$ does not depend on $a$. Therefore, we have that $\delta^{*}(n, m ; a) \leq \delta^{*}\left(n, m ; a^{\prime}\right)$ if $a \leq a^{\prime}$. That is, if it is optimal to moth-ball (or abandon) the project at a given $(\delta, n, m)$ and a given $a$, then this remains the case for all higher $a$. 


\section{Proof of Proposition 11}

The value of the firm given for this case by Proposition 4 is proportional to $\delta(t)$. This is the "cum-cash-flow" value. Let $C(t+1)$ denote the cash flow next period, which will be either zero or $-I(t+1)$. Both of these are proportional to $\delta(t+1)$. Accordingly,

$$
x(t+1) V[n(t+1), m(t+1), \delta(t+1)]=x(t+1) \delta(t+1) h[n(t+1), m(t+1)]
$$

Since, given time- $t$ information, the innovations in $x(t+1)$ and $\delta(t+1)$ are independent of those in $n(t+1)$ and $m(t+1)$, the current expectation of this value next period is

$$
E_{t}\{x(t+1) \delta(t+1) h[n(t+1), m(t+1)]\}=\pi_{y} \delta(t) \exp (\mu) E_{t}\{h[n(t+1), m(t+1)]\}
$$

The current (ex-cash-flow) value of the firm will then be

$E_{t}\left\{\frac{z(t+1)}{z(t)} x(t+1) \delta(t+1) h[n(t+1), m(t+1)]\right\}=\pi_{y} \delta(t) \exp (\mu-r-\lambda) E_{t}\{h[n(t+1), m(t+1)]\}$

because all the variables except $\delta(t+1)$ have innovations that, conditional on time- $t$ information, are independent of $z(t+1)$. Dividing the expected payoff, (55), by its value, (56), gives $e^{r+\lambda}$ as the per-dollar expected return.

\section{Value of the Project when Investment is Deterministic}

Lemma 3 When $\pi$ is known and the firm is has no option to suspend future investment, the value of the project is:

$$
V(n, \delta)=\alpha(n) \delta-\gamma(n)
$$

where

$$
\begin{gathered}
\alpha(n)=\frac{\chi^{N-n}}{(1-\beta)}-\frac{b}{1-(1-\pi) \beta}\left(\frac{1-\chi^{N-n}}{1-\chi}\right), \\
\gamma(n)=\left(\frac{a}{1-(1-\pi) \kappa}\right)\left(\frac{1-\psi^{N-n}}{1-\psi}\right),
\end{gathered}
$$

and where $\kappa \equiv \pi_{y} e^{-r}, \psi \equiv \frac{\pi \kappa}{1-(1-\pi) \kappa}$, and $\chi \equiv \frac{\pi \beta}{1-(1-\pi) \beta}$.

Proof: With no option component the value function just solves:

$$
V(n, \delta)=\pi V_{e}(n+1, \delta)+(1-\pi) V_{e}(n, \delta)-b \delta-a
$$


The conjecture that the solution has the form described in the proposition is verified simply through recursive substitution and simplification

\section{Proof of Proposition 12}

We prove this result for the case where the probability of technical success at each stage is known. Suppose the probabilities of success and of obsolescence over any interval of time, $\Delta$, are taken to be proportional to $\Delta$. Investment occurs at rate $I$, so the investment of time interval $\Delta$ is $I \Delta$. The cash flow at $t+\Delta, \delta(t+\Delta)$, is given by

$$
\delta(t) \exp \left[\left(\mu-\frac{1}{2} \sigma^{2}\right) \Delta+\sigma \sqrt{\Delta} \epsilon(t+\Delta)\right]
$$

where $\epsilon(t)$ is i.i.d. normal $(0,1)$. The continuously compounded riskless rate is $r$. As $\Delta$ goes to 0 , the process for $\delta(t)$ converges to a geometric Brownian motion. Our discrete-time model corresponds to $\Delta=1$. The probability of success over an interval of length $\Delta$ is taken to be $\pi \Delta$ and the probability of obsolescence is $a_{y} \Delta$ (so $\pi_{y}$ in the discrete-time model corresponds to $\left.\left(1-a_{y} \Delta\right)\right)$.

Under the risk neutral probability measure $(Q)$ implied by the pricing kernel we have assumed, the risk-neutral drift of $\delta(t)$ in the limiting case would be $\hat{\mu} \equiv \mu-\lambda$ and its volatility parameter would be unchanged.

The discrete-time valuation equation for stage $n$ is written as

$$
V(\delta, n)=\max \left[\left(1-a_{y} \Delta\right) A_{1},\left(1-a_{y} \Delta\right) A_{2}\right]
$$

where

$$
A_{1}=\pi \Delta \exp (-r \Delta) E_{t}^{Q} V(n+1, \delta(t+\Delta))+(1-\pi \Delta) \exp (-r \Delta) E_{t}^{Q} V[n, \delta(t+\Delta)]-I \Delta
$$

and

$$
A_{2}=\exp (-r \Delta) E_{t}^{Q} V[n, \delta(t+\Delta)]
$$

In the continuous time limit, $E_{t}^{Q} V[n+1, \delta(t+\Delta)]$ is equal to

$$
V(n+1, \delta(t))+V_{1}(n+1, \delta(t)) \hat{\mu} \delta(t) \Delta+\frac{1}{2} V_{11}(n+1, \delta(t)) \sigma_{\delta}^{2} \delta^{2}(t) \Delta+o(\Delta) .
$$

A similar expression describes $V(n, \cdot)$. Suppose that $V(n+1, \cdot)$ has already been obtained. The following derives a differential equation for $V(n, \cdot)$. In the region where research is continued, we have

$V(\delta, n)$ 


$$
=\pi V(n+1, \delta) \Delta+[1-\pi \Delta] \exp (-r \Delta)\left[1-a_{y} \Delta\right]\left[V(n, \delta)+V_{1}(n, \delta) \hat{\mu} \delta \Delta+\frac{1}{2} V_{11}(n, \delta) \sigma_{\delta}^{2} \delta^{2} \Delta\right]-I \Delta+o(\Delta) .
$$

Moreover, in the mothball region, we have

$$
\begin{aligned}
& V(\delta, n) \\
& \quad=\exp [-r \Delta]\left[1-a_{y} \Delta\right]\left[V(n, \delta)+V_{1}(n, \delta) \hat{\mu} \delta \Delta+\frac{1}{2} V_{11}(n, \delta) \sigma_{\delta}^{2} \delta^{2} \Delta\right]+o(\Delta) .
\end{aligned}
$$

Dividing both sides by $\Delta$ and taking limits, we find that in the continuation region, $V(n, \cdot)$ satisfies the following differential equation:

$$
V_{1}(n, \cdot) \hat{\mu} \delta+\frac{1}{2} V_{11}(n, \cdot) \sigma_{\delta}^{2} \delta^{2}-\left(r+a_{y}+\pi\right) V(n, \cdot)+\pi V(n+1, \cdot)-I=0,
$$

and in the mothball region, $V(n, \cdot)$ satisfies

$$
V_{1}(n, \cdot) \hat{\mu} \delta+\frac{1}{2} V_{11}(n, \cdot) \sigma_{\delta}^{2} \delta^{2}-\left(r+a_{y}\right) V(n, \cdot)=0 .
$$

We know that the continuation region is of the form $\delta \geq \delta^{*}(n)$. Thus, solving for $V(n, \cdot)$ involves solving for $\delta^{*}(n)$ and the above two differential equations, one holding for $\delta>\delta^{*}(n)$ and the other for $\delta<\delta^{*}(n)$. To determine $\delta^{*}(n)$ we would have to use value-matching (continuity of $V(n, \cdot)$ ) and smooth pasting (differentiability of $V(n, \cdot)$ ) conditions. For the result below, it is not necessary to know what $\delta^{*}(n)$ is, because our limiting result on risk premium is true for small $\delta$, i.e. when we are in the mothball region. In this region, the solution to the differential equation for $V(n, \cdot)$ is of the form $A(n) \delta^{\phi}$ where $\phi=\frac{-b+\sqrt{b^{2}+4 a c}}{2 a}$ with $a=.5 \sigma_{\delta}^{2}, b=\hat{\mu}-.5 \sigma_{\delta}^{2}$ and $c=\left(r+a_{y}\right)$. This, in turn, implies that in the entire mothball region, the risk premium on the $\mathrm{R} \& \mathrm{D}$ project is equal to $\phi \lambda\left(\right.$ since $\phi \lambda d t=-\operatorname{cov}_{t}\left(\frac{d V(n, \cdot)}{V(n, \cdot)}, \frac{d z}{z}\right)$ where $z(t)$ is the pricing kernel)

\section{Proof of Proposition 30}

Let $R_{t+1}(n, m)$ be the continuously compounded return from $t$ to $t+1$ of a project with $n$ completed stages and $m$ failures. Let the number of completed stages (failures) at time $t+1$ be denoted $n(t+1)(m(t+1))$. Then, conditional on survival $(x(t+1)=1)$,

$$
\begin{aligned}
R_{t+1}(n, m) & =\log \left(\frac{h(n(t+1), m(t+1)) \delta(t+1)}{h(n, m) \delta(t)}\right) \\
& =\log \left(\frac{h(n(t+1), m(t+1)) \delta(t)}{h(n, m) \delta(t)} \exp \left[\mu-\frac{\sigma_{\delta}^{2}}{2}+\sigma_{\delta} \epsilon_{\delta}(t+1)\right]\right) \\
& =K(n, m)+\log [h(n(t+1), m(t+1))]+\sigma_{\delta} \epsilon_{\delta}(t+1) .
\end{aligned}
$$

The result follows because the first term in the above expression is a constant and the last is a random variable with distribution $\mathrm{N}\left[0, \sigma_{\delta}\right]$. The middle term is a discrete random variable that 
takes on the value $\log [h(n+1, m)]$ with probability $\bar{\pi}(n, m)$ and the value $\log [h(n, m+1)]$ with probability $1-\bar{\pi}(n, m)$.

\section{B Numerical Details}

Our programs solve numerically for the value of the $\mathrm{R} \& \mathrm{D}$ venture at each $n, m$ and over a range of discrete values for $\delta$. The solution technique we use is to recursively solve (12) backwards starting with the value of the completed project and the value of an abandoned project, both of which are known analytically. Specifically, $V(N, \cdot, \delta)$ is given by Proposition 1. Proposition 6 is used to derive $m^{*}(n)$ for all values of $n$. Using Proposition 10, we have that for all $m>m^{*}(n)$ and $n<N$, $V(n, m, \delta)=0 . V\left(N-1, m^{*}(N-1), \delta\right)$ can then be implicitly defined using (12):

$$
V\left(N-1, m^{*}(N-1), \delta\right)=\max \left\{\bar{\pi}(n, m) V_{e}(N, \cdot, \delta), V_{e}\left(N-1, m^{*}(N-1), \delta\right)\right\}
$$

This equation is then solved numerically by first initially guessing the value of $V\left(N-1, m^{*}(N-1), \delta\right)$ over a discrete set of values (grid points) for $\delta$. Values between these grid points are derived by quadratic interpolation. Using this guess a new value of $V\left(N-1, m^{*}(N-1), \delta\right)$ at each grid point is derived and this value is used as the new input. This process is repeated until the value over the grid points converge (the new value is no more than $0.001 \%$ different from the old value anywhere in the grid). This process is then repeated for all remaining values of $m<m^{*}(N-1)$. Once this is complete, one more step back can be taken and the value for $V\left(N-2, m^{*}(N-2), \delta\right)$ can be derived using the same process. This is continued until $V$ is derived for all $n$ and $m$ at every grid point. The only remaining issue is what is done when the value is needed for a $\delta$ outside of the grid. Since our grids start at 0 , this only occurs for values of $\delta$ larger than the maximum grid value. Since for large $\delta$, the mothball option value is small, so we use the value of the project with no option which can be solved for analytically. The following proposition, which is stated without proof, gives this solution.

Proposition 14 If there is no option to halt development (so that unless the project is extinguished through obsolescence, the investment is required to be made each period) then the value function is

$$
V(n, m, \delta)=\hat{h}(n, m) \delta+\hat{g}(n, m)
$$

so

$$
V_{e}(n, m, \delta)=\hat{h}(n, m) \beta \delta+\kappa \hat{g}(n, m)
$$

where $\hat{h}(n, m)$ and $\hat{g}(n, m)$, stated recursively, are

$$
\begin{aligned}
\hat{h}(n, m) & =\bar{\pi}(n, m) \beta \hat{h}(n+1, m)+(1-\bar{\pi}(n, m)) \beta \hat{h}(n, m+1)-b \\
\hat{g}(n, m) & =\bar{\pi}(n, m) \kappa \hat{g}(n+1, m)+(1-\bar{\pi}(n, m)) \kappa \hat{g}(n, m+1)-a
\end{aligned}
$$


with boundary conditions $\hat{h}\left(n, m^{*}(n)+1\right)=\hat{g}\left(n, m^{*}(n)+1\right)=\hat{g}(N, \cdot)=0$ and $\hat{h}(N, \cdot)=g(N)$.

In our simulations we use a 200 point grid between the points 0 and 100 . The spacing between the points was not equal, more points were used at lower values of $\delta$. The ratio between the largest spacing between grid points and the smallest was 10 . 


\section{References}

Berk, J., R. C. Green and V. Naik (1997), "Optimal Investment, Growth Options and Security Returns," Working Paper, School of Business Administration, University of Washington.

Bhattacharya, S. and D. Mookherjee (1986), "Portfolio Choice in Research and Development," Rand Journal of Economics, 17:594-605.

Brealey, R. A. and S. C. Myers (1996), Principles of Corporate Finance, McGraw-Hill, Inc.

Brealey, R. A., S. C. Myers and A. J. Marcus (1995), Fundamentals of Corporate Finance, McGrawHill, Inc.

Childs, P. D. and A. J. Triantis (1997), "Dynamic R\&D Investment Policies," Working Paper, College of Business and Management, University of Maryland.

Dasgupta, P. and E. Maskin (1987), "The Simple Economics of Research Portfolios," The Economic Journal, 97:581-595.

M. H. DeGroot (1970), Optimal Statistical Decisions, McGraw-Hill, Inc.

Dutta, P. K. (1997), "Optimal management of an R\&D budget," Journal of Economic Dynamics and Control, 21:575-602.

Grossman, G. M. and C. Shapiro (1986), "Optimal dynamic R\&D programs," Rand Journal of Economics, 17:581-593.

Gruver, G. W. (1991), "Optimal R\&D policy for a patent race with uncertain duration," Mathematical Social Sciences, 22:69-85.

Majd, S. and R. S. Pindyck (1987), "Time to Build, Option Value, and Investment Decisions," Journal of Financial Economics, 18:7-27.

Myers, S. C. and C. D. Howe (1997), "A Life-Cycle Model of Pharmaceutical R\&D," Technical Report, Program on the Pharmaceutical Industry, Massachusetts Institute of Technology.

Pakes, A., "Patents as Options: Some Estimates of the Value of Holding European Patent Stocks," Econometrica, 54:755-784.

Pindyck, R. S. (1993), "Investments of Uncertain Cost," Journal of Financial Economics, 34:53-76.

Posner, M. J. M. and D. Zuckerman, "Optimal R\&D Programs in a Random Environment," Journal of Applied Probability, 27:343-350.

Rockafellar, R. T. (1970), Convex Analysis, Princeton University Press. 
Schwartz, E. S. and M. Moon (1995), "Evaluating Research and Development investments," Working Paper, Anderson Graduate School of Management at UCLA.

Weitzman, M. L. (1979), "Optimal Search for the Best Alternative," Econometrica, 47:641-654.

Weitzman, M., W. Newey and M. Rabin (1981), "Sequential R\&D Strategy for Synfuels," Bell Journal of Economics, 12:574-590. 